\title{
Classical provability of uniform versions and intuitionistic provability
}

\author{
Makoto Fujiwara* \\ Mathematical Institute \\ Tohoku University \\ 6-3, Aramaki Aoba, Aoba-ku \\ Sendai, Miyagi, Japan \\ sb0m29@math.tohoku.ac.jp
}

\author{
Ulrich Kohlenbach ${ }^{\dagger}$ \\ Fachbereich Mathematik \\ TU Darmstadt, Schlossgartenstraße7 \\ D-64289 Darmstadt, Germany \\ kohlenbach@mathematik.tu-darmstadt.de
}

August 17, 2013

\begin{abstract}
Along the line of Hirst-Mummert [9] and Dorais [4], we analyze the relationship between the classical provability of uniform versions Uni(S) of $\Pi_{2}$-statements $S$ with respect to higher order reverse mathematics and the intuitionistic provability of $S$. Our main theorem states that (in particular) for every $\Pi_{2}$-statement $S$ of some syntactical form, if its uniform version derives the uniform variant of ACA over a classical system of arithmetic in all finite types with weak extensionality, then $\mathrm{S}$ is not provable in strong semi-intuitionistic systems including bar induction BI in all finite types but also nonconstructive principles such as König's lemma KL and uniform weak König's lemma UWKL. Our result is applicable to many mathematical principles whose sequential versions imply ACA.
\end{abstract}

\section{Introduction}

Classical uniform provability is closely related to intuitionistic provability. In the practice of reverse mathematics [22], sequential versions of $\Pi_{2}^{1}$-theorems, which assert to solve infinitely many instances of a particular problem simultaneously, have been investigated in order to reveal the lack of uniformity of the proof of $S$ in RCA. It has been recently established in [9] and [4] that for $\Pi_{2}^{1}$-statements of some syntactical form, the provability in certain (semi-)intuitionistic systems guarantees the sequential provability in RCA (or +WKL). Such kind of results are called "uniformization theorems". Uniformization theorems allow us to use the sequential reverse mathematics to demonstrate the unprovability of several mathematical principles in (semi-)intuitionistic systems. The following table 1 gives the picture of existing uniformization theorems on sequential versions. As shown in [4], not only the uniformization theorem for RCA but also the uniformization theorem for RCA + WKL holds in a second-order setting. Now, what can we show in a higher-order (all finite types) setting? Our main interest in this paper is in the relationship between classical uniform provability and intuitionistic provability with respect to WKL in a higher-order setting $([\star]$ in table 1$)$.

\footnotetext{
${ }^{*}$ The 1 st author has been supported by Shigakukai.

${ }^{\dagger}$ The 2nd author has been supported by the German Science Foundation (DFG Project KO 1737/5-2).
} 


\begin{tabular}{c|c|c} 
& Higher-order setting & Second-order setting \\
\hline RCA + WKL & {$[\star]$} & Dorais [4, Corollary 3.9] \\
\hline RCA & Hirst-Mummert [9, Theorem 3.6 \& Theorem 5.6] & Dorais [4, Corollary 2.9]
\end{tabular}

Table 1: Uniformization theorems

In fact, there is a stronger form to capture uniform provability than just sequentialization. For a sentence $\mathrm{S}:=\forall X(A(X) \rightarrow \exists Y B(X, Y))$, one can consider a sentence

$$
\exists F \forall X(A(X) \rightarrow B(X, F(X))),
$$

which states the existence of a uniform procedure $F$ to construct a solution for each problem $X$. Throughout this paper, we call this sentence the uniform version of $\mathrm{S}$ and denote it as Uni( $\mathrm{S})$. This is the fully uniform version of $S$ compared to the sequential version which is only a weaker representation of uniformity. However, for a $\Pi_{2}^{1}$-sentence, this uniform version is not naturally represented in the language of second-order arithmetic since $F$ is a third-order object. To treat full uniform versions, we use systems of arithmetic in all finite types. Uniform versions of ordinary mathematical theorems have been investigated in the context of higher order reverse mathematics $[18,21]$.

In Section 3, we show not only the uniformization theorem on uniform versions for RCA but also that for RCA + WKL (positioned at $[\star]$ in table 1 ). The proof is respectively based on modified realizability and the monotone Dialectica interpretation with the use of the technique of elimination of extensionality. The uniformization results on sequential versions in [9] are the immediate corollaries of our results since the sequential version follows from the uniform version.

In Section 4, we show a related metatheorem which states that (in particular) for every $\Pi_{2}$-statement $S$ of some syntactical form, if its uniform version Uni(S) derives the uniform variant $\left(\exists^{2}\right)$ of ACA over a classical higher-order system with weak extensionality, then $\mathrm{S}$ is not provable in extremely strong semi-intuitionistic systems $\mathscr{T}$ which include bar induction BI in all types but also weak König's lemma WKL and even uniform weak König's lemma UWKL and König's lemma KL. In particular, $\mathscr{T}$ is strong enough (even without UWKL and KL) to interpret classical analysis with full dependent choice via negative translation. That is, by applying this metatheorem, one can obtain stronger unprovability results than what follows from uniformization theorems. In this sense, one can think of our metatheorem as an extended variant of the higher-order uniformization theorem positioned at $[\star]$ in table 1 . In the proof, we use (a variant of) the Dialectica interpretation, negative translation, a nonstandard axiom $F^{-}$and the model of all strongly majorizable functionals.

Roughly speaking the metatheorem often allows one to detect using classical reasoning on Uni(S) that $S$ intuitionistically implies at least the $\Pi_{1}^{0}$-law-of-excluded-middle principle $\Pi_{1}^{0}$-LEM (and so - in the presence of Markov's principle - $\Sigma_{1}^{0}$-LEM) rather than only the strictly weaker principle $\Sigma_{1}^{0}$-LLPO (as WKL already does; see [1] for details on these principles).

In Section 5, we demonstrate that our metatheorem in Section 4 is applicable to concrete mathematical principles to show, using classical reasoning on the uniform versions of principles $S$ only, the unprovability of $\mathrm{S}$ in the semi-intuitionistic systems mentioned above. The investigation of the strength of uniform versions in higher order reverse mathematics plays an important role in the application of our metatheorem. Roughly speaking, our metatheorem is applicable to statements whose sequential versions imply ACA.

We assume that the reader is familiar with intuitionistic and classical arithmetic in all finite types (see [6, 24, 19] for basic knowledge). Most of the technical tools used in this paper can be found in [19]. 


\section{Axiom systems}

We use systems of higher-order arithmetic (e.g., E-HA ${ }^{\omega}, \mathrm{E}_{\mathrm{PA}}{ }^{\omega}$ ) formulated in the language of functionals in all finite types as the framework for our analysis. Here, by 'higher-order', we do not mean a genuine higher order theory but a many-sorted first order theory with variables for functionals of all finite types. We employ the type notation from [19]. Note that the superscripts on quantified variables indicate their type and $x^{\rho}$ denotes the tuple of variables with corresponding types. In addition, $\mathbf{T}$ is the set of all types. We recall that a type- 0 functional is a natural number, a type-1 $(=0(0))$ functional is a function from natural numbers to natural numbers, and a type-2 $(=0(1))$ functional is a functional from type 1 functionals to natural numbers. In addition, we refer the reader to [19, Chapter 3] for the formal definitions of (subsystem of) higher-order intuitionistic arithmetics $\mathrm{E}-\mathrm{HA}{ }^{\omega}, \widehat{\mathrm{E}-\mathrm{HA}}^{\omega} \uparrow$ and (subsystem of) higher-order classical arithmetics E-PA ${ }^{\omega}, \widehat{\mathrm{E}-\mathrm{PA}}^{\omega} \uparrow$. Note that E-HA ${ }^{\omega}$ is the finite type extension of $\mathrm{HA}$ with full extensionality, and that $\mathrm{E}-\mathrm{PA}{ }^{\omega}$ is the finite type extension of PA with full extensionality. Furthermore, we use the weakly extensional systems $W E-H A^{\omega}, \widehat{W_{E}-H A}{ }^{\omega} \uparrow, W E-P A^{\omega}$ and $\widehat{\mathrm{WE}-\mathrm{PA}}^{\omega} \uparrow$ (see further below as well as $\left[19\right.$, Chapter 3]) corresponding to E-HA ${ }^{\omega}, \widehat{\mathrm{E}}-\mathrm{HA}^{\omega} \uparrow, \mathrm{E}-\mathrm{PA}{ }^{\omega}$ and $\widehat{\mathrm{E}-\mathrm{PA}}^{\omega} \uparrow$ respectively. $\widehat{\mathrm{E}-\mathrm{HA}}^{\omega} \uparrow$ (resp. $\widehat{\mathrm{WE}-\mathrm{HA}}^{\omega} \uparrow, \widehat{\mathrm{E}-\mathrm{PA}}^{\omega} \uparrow, \widehat{\mathrm{WE}-\mathrm{PA}}^{\omega} \uparrow$ ) is Feferman's ([6]) restriction of E-HA${ }^{\omega}$ (resp. WE-HA ${ }^{\omega}, \mathrm{E}_{-\mathrm{PA}}{ }^{\omega}, \mathrm{WE}^{\mathrm{P}} \mathrm{A}^{\omega}$ ) to primitive recursion of type 0 and quantifier-free induction.

We first recall the definitions of key principles in this paper (cf. $[19,16,18])$.

- $\left(\exists^{2}\right): \exists E^{2} \forall f^{1}\left(E f=0 \leftrightarrow \exists x^{0}(f x=0)\right)$.

- $\left(\mu^{2}\right): \exists \mu^{2} \forall f^{1}\left(\exists x^{0}(f x=0) \rightarrow f(\mu f)=0\right)$.

- WKL (weak König's lemma): $\forall f^{1}\left(T^{\infty}(f) \rightarrow \exists b \leq_{1} \lambda k .1 \forall x^{0}(f(\bar{b} x)=0)\right)$, where $T^{\infty}(f)$ expresses that $f$ represents an infinite binary tree.

- UWKL (uniform weak König's lemma): $\exists \Phi \leq_{1(1)} 1 \forall f^{1}\left(T^{\infty}(f) \rightarrow \forall x^{0}(f((\overline{\Phi f}) x)=0)\right)$.

Note that $\left(\exists^{2}\right)$ is the uniform variant of ACA and UWKL is the uniform version of WKL. Next we recall the definitions of axiom schemes used in this paper.

Remark 2.1. In this paper, we define the axioms with single variables for simplicity. Note that the version with single variables $\left(x^{\rho}, y^{\tau}\right.$ etc.) implies the one with tuples $\left(\underline{x}^{\rho}, y^{\underline{\tau}}\right.$ etc.) since one can show in WE-HA ${ }^{\omega}$ that finite tuples of variables of different types can be coded together into a single variable (see e.g. [24] for details).

For the rest of the present paper $A_{0}, B_{0}$ etc. always denote quantifier-free formulas. ' $\operatorname{deg}(\rho)$ ' denotes the usual degree of the type $\rho$, i.e. $\operatorname{deg}(0):=0, \operatorname{deg}(\rho(\tau)):=\max \{\operatorname{deg}(\tau)+1, \operatorname{deg}(\rho)\}$.

In addition, the formulas in the following schemes may have parameters of arbitrary types.

- $\mathrm{AC}^{\rho, \tau}: \forall x^{\rho} \exists y^{\tau} A(x, y) \rightarrow \exists Y^{\tau \rho} \forall x^{\rho} A(x, Y x)$.

$\mathrm{AC}:=\bigcup_{\rho, \tau \in \mathbf{T}}\left\{\mathrm{AC}^{\rho, \tau}\right\}$ (axiom scheme of choice).

$\mathrm{AC}^{0}:=\bigcup_{\tau \in \mathbf{T}}\left\{\mathrm{AC}^{0, \tau}\right\}$ (axiom scheme of countable choice).

- $\mathrm{AC} !^{\rho, \tau}: \forall x^{\rho} \exists y^{\tau}\left(A(x, y) \wedge \forall z^{\tau}(A(x, z) \rightarrow y=z)\right) \rightarrow \exists Y^{\tau \rho} \forall x^{\rho} A(x, Y x)$.

$\mathrm{AC} !^{1}:=\bigcup_{\rho, \tau \in \mathbf{T}, \operatorname{deg}(\rho) \leq 1}\left\{\mathrm{AC} !^{\rho, \tau}\right\}$ (axiom scheme of unique choice for functions). 
- $\mathrm{QF}-\mathrm{AC}^{\rho, \tau}$ is the restriction of $\mathrm{AC}^{\rho, \tau}$ to quantifier-free $A$.

$\mathrm{QF}-\mathrm{AC}:=\bigcup_{\rho, \tau \in \mathbf{T}}\left\{\mathrm{QF}-\mathrm{AC}{ }^{\rho, \tau}\right\}$ (quantifier-free axiom of choice).

- $\mathrm{IP}_{\mathrm{ef}}^{\rho}:\left(A_{\mathrm{ef}} \rightarrow \exists x^{\rho} B(x)\right) \rightarrow \exists x^{\rho}\left(A_{\mathrm{ef}} \rightarrow B\left(x^{\rho}\right)\right)$, where $A_{\mathrm{ef}}$ is $\exists$-free (i.e. $A_{\mathrm{ef}}$ does not contain $\exists, \vee$ ) and does not contain $x$ free.

$\operatorname{IP}_{\mathrm{ef}}^{\omega}:=\bigcup_{\rho \in \mathbf{T}}\left\{\operatorname{IP}_{\mathrm{ef}}^{\rho}\right\}$ (independence-of-premise schema for $\exists$-free premises).

- $\mathrm{IP}_{\neg}^{\rho}:\left(\neg A \rightarrow \exists x^{\rho} B(x)\right) \rightarrow \exists x^{\rho}\left(\neg A \rightarrow B\left(x^{\rho}\right)\right)$, where $A$ does not contain $x$ free.

$\operatorname{IP}_{\neg}^{\omega}:=\bigcup_{\rho \in \mathbf{T}}\left\{\operatorname{IP}_{\neg}^{\rho}\right\}$ (independence-of-premise schema for negated premises).

- $\operatorname{IP}_{\forall}^{\rho, \tau}:\left(\forall u^{\tau} A_{0}(u) \rightarrow \exists x^{\rho} B(x)\right) \rightarrow \exists x^{\rho}\left(\forall u^{\tau} A_{0}(u) \rightarrow B(x)\right)$, where $A_{0}$ does not contain $x$ free.

$\operatorname{IP}_{\forall}^{\omega}:=\bigcup_{\rho, \tau \in \mathbf{T}}\left\{\operatorname{IP}_{\forall}^{\rho, \tau}\right\}$ (independence-of-premise schema for universal premises).

$\operatorname{IP}_{\forall}^{\leq 1, \leq 1}$ is the restriction of $\operatorname{IP}_{\forall}^{\omega}$ types $\rho$ and $\tau$ of degree $\leq 1$ (note that $B$ may contain other variables of arbitrary types).

- $\mathrm{M}^{\rho}:=\neg \neg \exists x^{\rho} A_{0}(x) \rightarrow \exists x^{\rho} A_{0}(x)$

$\mathrm{M}^{\omega}:=\bigcup_{\rho \in \mathbf{T}} \mathrm{M}^{\rho}$ (Markov's principle in finite types).

$\mathbf{M}^{\leq 1}$ is the restriction of $\mathbf{M}^{\omega}$ to types $\rho$ of degree $\leq 1$.

Definition 2.2. (See also $[18,9])$

1. $\mathrm{RCA}_{0}^{\omega}:=\widehat{\mathrm{E}-\mathrm{PA}}^{\omega} \uparrow+\mathrm{QF}-\mathrm{AC}^{1,0}$.

2. $\mathrm{RCA}^{\omega}:=\mathrm{E}^{-\mathrm{PA}}{ }^{\omega}+\mathrm{QF}-\mathrm{AC}^{1,0}$.

3. $\mathrm{WRCA}_{0}^{\omega}:=\widehat{\mathrm{WE}-\mathrm{PA}}^{\omega} \uparrow+\mathrm{QF}-\mathrm{AC}^{1,0}$.

4. $\mathrm{WRCA}^{\omega}:=\mathrm{WE}^{\mathrm{PA}}{ }^{\omega}+\mathrm{QF}-\mathrm{AC}^{1,0}$.

Note that $\mathrm{RCA}_{0}^{\omega}$ and $\mathrm{RCA}^{\omega}$ have the full extensionality axiom

$$
\text { (E) : } \bigcup_{\rho=0 \rho_{1} \ldots \rho_{k}, \rho_{i} \in \mathbf{T}} \forall z^{\rho}, x_{1}^{\rho_{1}}, y_{1}^{\rho_{1}}, \ldots, x_{k}^{\rho_{k}}, y_{k}^{\rho_{k}}\left(\bigwedge_{i=1}^{k}\left(x_{i}=\rho_{i} y_{i}\right) \rightarrow z \underline{x}={ }_{0} z \underline{y}\right) .
$$

Here, for types $\tau=0 \tau_{n} \ldots \tau_{1}, x=\tau y$ is defined as extensional equality: $\forall v_{1}^{\tau_{1}}, \ldots, v_{n}^{\tau_{n}}\left(x \underline{v}={ }_{0} y \underline{v}\right)$. On the other hand, $\mathrm{WRCA}_{0}^{\omega}$ and WRCA ${ }^{\omega}$ have only a quantifier-free rule of extensionality

$$
(\mathrm{QF}-\mathrm{ER}): \frac{A_{0} \rightarrow s={ }_{\rho} t}{A_{0} \rightarrow r^{\tau}\left[s / x^{\rho}\right]=\tau r\left[t / x^{\rho}\right]}
$$

instead of the full extensionality axiom (E).

$\mathrm{RCA}_{0}^{\omega}$ has been used as the base system for higher order reverse mathematics so far [18]. In Section 5 , however, we will investigate the uniform versions of several principles over $\mathrm{WRCA}_{0}^{\omega}$ to apply our main metatheorem. 
Warning. $\mathrm{RCA}_{0}^{\omega}$ and $\mathrm{RCA}^{\omega}$ satisfy the deduction theorem, while $\mathrm{WRCA}_{0}^{\omega}$ and $\mathrm{WRCA}^{\omega}$ do not satisfy the deduction theorem as follows from [15].

Proposition $2.3([18,9])$. 1. $\mathrm{RCA}_{0}^{\omega}$ and $\mathrm{WRCA}_{0}^{\omega}$ are conservative extensions of $\mathrm{RCA}_{0}^{2}:=\widehat{\mathrm{E}-\mathrm{PA}}^{2} \uparrow+\mathrm{QF}-\mathrm{AC}^{0,0}$, which is the function-based second-order system equivalent to the set-based second-order system $\mathrm{RCA}_{0}$ in ordinary reverse mathematics.

2. $\mathrm{RCA}^{\omega}$ and $\mathrm{WRCA}^{\omega}$ are conservative extensions of $\mathrm{RCA}^{2}:=\mathrm{E}-\mathrm{PA}^{2}+\mathrm{QF}-\mathrm{AC}^{0,0}$, which is the functionbased second-order system equivalent to the set-based second-order system RCA (having full induction scheme) in ordinary reverse mathematics.

\section{Uniformization theorems}

\subsection{Uniformization theorem for RCA}

Definition 3.1 ([24, 19,9]). 1. A formula of $\mathscr{L}\left(\mathrm{E}^{-H A} A^{\omega}\right)$ (or $\mathscr{L}\left(\widehat{\mathrm{E}-\mathrm{HA}}^{\omega} \uparrow\right)$ in context) is $\exists$-free if it is built up from prime formulas by means of $\wedge, \rightarrow$ and $\forall$ only.

2. $\Gamma_{1}$ is the syntactic class of formulas of $\mathscr{L}\left(\mathrm{E}^{-\mathrm{HA}}{ }^{\omega}\right)$ (or $\mathscr{L}\left(\widehat{\mathrm{E}-\mathrm{HA}}^{\omega} \uparrow\right)$ in context) defined inductively as follows.

(a) Prime formulas are in $\Gamma_{1}$.

(b) If $A, B$ are in $\Gamma_{1}$, then $A \wedge B, A \vee B, \forall x A(x), \exists x A(x)$ are in $\Gamma_{1}$.

(c) If $A$ is $\exists$-free and $B \in \Gamma_{1}$, then $(\exists \underline{x} A \rightarrow B) \in \Gamma_{1}$.

Proposition 3.2. 1. Let $A$ be an arbitrary formula of $\mathscr{L}\left(\mathrm{E}^{-\mathrm{H}} \mathrm{A}^{\omega}\right)$. Then one can construct an $\exists$-free formula $B_{\text {ef }}$ such that

$$
\mathrm{E}-\mathrm{HA}^{\omega}+\mathrm{AC}+\mathrm{IP}_{\mathrm{ef}}^{\omega} \vdash \neg A \leftrightarrow B_{\mathrm{ef}} .
$$

2. Let $A_{\mathrm{ef}}$ be an $\exists$-free formula of $\mathscr{L}\left(\mathrm{E}-\mathrm{HA}{ }^{\omega}\right)$. Then

$$
\mathrm{E}-\mathrm{HA}^{\omega} \vdash A_{\mathrm{ef}} \leftrightarrow \neg \neg A_{\mathrm{ef}} .
$$

These also hold for $\widehat{\mathrm{E}-\mathrm{HA}}^{\omega} \uparrow$ instead of $\mathrm{E}-\mathrm{HA}^{\omega}$.

Proof. 1. See [19, Proposition 5.14]. 2. One can show E-HA ${ }^{\omega} \vdash \neg \neg A_{\mathrm{ef}} \rightarrow A_{\mathrm{ef}}$ by easy induction on the structure of $A_{\text {ef }}$ with the use of the fact that prime formulas are decidable. The opposite direction is obvious. The same proof also works for the analogous result for $\widehat{\mathrm{E}-\mathrm{HA}}^{\omega} \uparrow$.

As corollary to Proposition 3.2, we get that $\mathrm{IP}_{\mathrm{ef}}^{\omega}$ and $\mathrm{IP}_{\neg}^{\omega}$ are equivalent in the presence of AC.

Corollary 3.3. 1. $\mathrm{E}-\mathrm{HA}{ }^{\omega}+\mathrm{AC} \vdash \mathrm{IP}_{\mathrm{ef}}^{\omega} \rightarrow \mathrm{IP}_{\neg}^{\omega}$.

2. $\mathrm{E}_{-} \mathrm{HA}^{\omega} \vdash \mathrm{IP}_{\neg}^{\omega} \rightarrow \mathrm{IP}_{\mathrm{ef}}^{\omega}$.

We first show a uniformization theorem for uniform versions via modified realizability. The proof is essentially the same as for the main theorem of Hirst and Mummert [9, Theorem 3.6]. 
Proposition 3.4. Let $\Delta_{\mathrm{ef}}^{\mathrm{RCA}}{ }^{\omega}$ be the class of $\exists$-free sentences provable in $\mathrm{RCA}^{\omega}$. For a sentence $\mathrm{S}:=\forall x^{\rho}(A(x) \rightarrow$ $\left.\exists y^{\tau} B(x, y)\right)$ of $\mathscr{L}\left(\mathrm{E}-\mathrm{HA}^{\omega}\right)$ where $A(x)$ is $\exists$-free and $B(x, y)$ is in $\Gamma_{1}$, if

$$
\mathrm{E}-\mathrm{HA}{ }^{\omega}+\mathrm{AC}+\mathrm{IP}_{\mathrm{ef}}^{\omega}+\Delta_{\mathrm{ef}}^{\mathrm{RCA}}+\mathrm{S},
$$

then

$$
\mathrm{RCA}^{\omega} \vdash \operatorname{Uni}(\mathrm{S}) \text {. }
$$

This also holds for $\widehat{\mathrm{E}-\mathrm{HA}}^{\omega} \uparrow$ and $\mathrm{RCA}_{0}^{\omega}$ instead of ${\mathrm{E}-\mathrm{HA}^{\omega}}^{\omega}$ and $\mathrm{RCA}^{\omega}$.

Proof. Since $A(x)$ is $\exists$-free, $\forall x \exists y(A(x) \rightarrow B(x, y))$ is provable in $\mathrm{E}^{-\mathrm{HA}^{\omega}}+\mathrm{AC}+\mathrm{IP}_{\mathrm{ef}}^{\omega}+\Delta_{\mathrm{ef}}^{\mathrm{RCA}}$. Note that $\forall x \exists y(A(x) \rightarrow B(x, y))$ is in $\Gamma_{1}$ since $B(x, y)$ is in $\Gamma_{1}$. Then one can construct a closed term $t$ such that $\mathrm{E}-\mathrm{HA}^{\omega}+$ $\Delta_{\mathrm{ef}}^{\mathrm{RC} \mathrm{A}^{\omega}} \vdash \forall x(A(x) \rightarrow B(x, t x))$ by using modified realizability interpretation as in the proof of [9, Lemma 3.5] (actually using [19, Theorem 5.8 and Lemma 5.20]). Since RCA ${ }^{\omega}$ is an extension of $\mathrm{E}-\mathrm{HA} A^{\omega}+\Delta_{\mathrm{ef}}^{\mathrm{RCA}}{ }^{\omega}$, we have $\mathrm{RCA}^{\omega} \vdash \mathrm{Uni}(\mathrm{S})$. The same proof works for the analogous result for $\widehat{\mathrm{E}-\mathrm{HA}}^{\omega} \uparrow$ and $\mathrm{RCA}_{0}^{\omega}$.

Warning. Proposition 3.4 does not hold for every sentence $\forall x^{\rho}\left(A(x) \rightarrow \exists y^{\tau} B(x, y)\right)$ in $\Gamma_{1}$. In fact, $\forall f^{1}\left(\exists y^{1}(f y=\right.$ $\left.0) \rightarrow \exists x^{1}(f x=0)\right) \in \Gamma_{1}$ is logically valid, but its uniform version $\left(\mu^{2}\right)$ is not provable in $\operatorname{RCA}^{\omega}$. This means that it is essential to restrict the syntactical form of $A(x)$ to $\exists$-free in Proposition 3.4.

Remark 3.5. All sentences $\forall x^{\rho} \exists y^{\tau} A_{0}(x, y)$ provable in $\mathrm{RCA}^{\omega}$, where the degree of the type $\rho$ is not greater than 1 , the type $\tau$ is arbitrary and $A_{0}$ is quantifier-free, are included in $\mathrm{E}-\mathrm{HA}^{\omega}+\mathrm{AC}+\mathrm{IP}_{\mathrm{ef}}^{\omega}+\Delta_{\mathrm{ef}}^{\mathrm{RCA}}$, since one can show $\mathrm{WE}-\mathrm{HA}^{\omega} \vdash \forall x A_{0}(x, t x)\left(\in \Delta_{\mathrm{ef}}^{\mathrm{RCA}^{\omega}}\right)$ via elimination of extensionality (Lemma 3.14), negative translation (Lemma 4.6) and the Dialectica interpretation ([19, Theorem 8.6]).

While the proof of Proposition 3.4 is based on modified realizability interpretation, by using modified realizability interpretation with truth instead (actually using [19, Theorem 5.23 and Lemma 5.6] instead of [19, Theorem 5.8 and Lemma 5.20] in the proof of Proposition 3.4), one can show that for a sentence $\mathrm{S}:=$ $\forall x(\neg A(x) \rightarrow \exists y B(x, y))$ of $\mathscr{L}\left(\mathrm{E}-\mathrm{HA}^{\omega}\right)$ where $A(x)$ is arbitrary, if

$$
\mathrm{E}-\mathrm{HA}{ }^{\omega}+\mathrm{QF}-\mathrm{AC}^{1,0}+\mathrm{IP}_{\neg}^{\omega}+\Delta_{\neg}^{\mathrm{RCA}^{\omega}} \vdash \mathrm{S}
$$

then

$$
\left.\mathrm{RCA}^{\omega} \text { (actually E-HA }{ }^{\omega}+\mathrm{QF}-\mathrm{AC}^{1,0}+\mathrm{IP}_{\neg}^{\omega}+\Delta_{\neg}^{\mathrm{RCA}^{\omega}}\right) \vdash \mathrm{Uni}(\mathrm{S}),
$$

where $\Delta_{\neg}^{\mathrm{RCA}}{ }^{\omega}$ be the class of negated sentences provable in RCA ${ }^{\omega}$. Compared to Proposition 3.4, the syntactical restriction of $B(x, y)$ is dropped. In addition, since we don't have $\mathrm{AC}$ now, $\mathrm{IP}_{\neg}^{\omega}$ and $\Delta_{\neg}^{\mathrm{RCA}}{ }^{\omega}$ seem to be proper extensions of $\mathrm{IP}_{\mathrm{ef}}^{\omega}$ and $\Delta_{\mathrm{ef}}^{\mathrm{RCA}}{ }^{\omega}$ respectively.

\subsection{Uniformization theorem for $\mathrm{RCA}+\mathrm{WKL}$}

As for Proposition 3.4, one can also show another uniformization theorem on uniform versions via the Dialectica interpretation, which is the counterpart of [9, Theorem 5.6]. In this case, the syntactic class involved is restricted little more than in Proposition 3.4 and the base system is weakened to the weakly extensional one, but the non-intuitionistic scheme $\mathrm{M}^{\omega}$ can be added to the system in the assumption. (Note that WE-HA ${ }^{\omega} \subseteq \mathrm{E}-\mathrm{HA}{ }^{\omega}$ and $\widehat{W E}-H A^{\omega} \uparrow \subseteq \widehat{E}-H A^{\omega} \uparrow$.) Furthermore, in the sense of applications to sequential reverse mathematics, one can extend this result so that the system in the assumption includes WKL and even König's lemma KL, whereas the 
classical system in the conclusion contains UWKL. Such an extension (in the absence of $\mathbf{M}^{\omega}$ ) is also possible for Proposition 3.4, but the result would be less meaningful as in the presence of extensionality UWKL already proves uniform arithmetical comprehension $\left(\exists^{2}\right)$ (see [16, Proposition 3.4]) while UWKL is still weak relative to WRCA $^{\omega}$ (see again [16]).

The following syntactical form is important in our results.

Definition 3.6 ([13]). $\Delta$ denotes a set of sentences of the form

$$
\forall a^{\delta} \exists b \leq_{\sigma} r a \forall c^{\gamma} B_{0}(a, b, c),
$$

where $B_{0}(a, b, c)$ is quantifier-free and does not contain any further free variables than those shown, $r$ is a closed term (of suitable type) of E-HA ${ }^{\omega}$ (or $\widehat{\mathrm{E}-\mathrm{HA}}^{\omega} \uparrow$ in context), the types $\delta, \sigma, \gamma$ are arbitrary, and ' $b \leq_{\sigma} r a$ ' is defined pointwise, i.e $x \leq_{\sigma} y:=\forall \underline{v}\left(x \underline{v} \leq_{0} y \underline{v}\right)$.

Moreover, $\widetilde{\Delta}$ denotes a corresponding set of the Skolem normal forms of the sentences in $\Delta$

$$
\left\{\widetilde{\mathrm{T}}: \equiv \exists B \leq r \forall a \forall c B_{0}(a, B a, c): \mathrm{T}: \equiv \forall a^{\delta} \exists b \leq_{\sigma} r a \forall c^{\gamma} B_{0}(a, b, c) \in \Delta\right\} .
$$

Throughout this paper, for a sentence $\mathrm{T}$ of the form of $\Delta$, we denote the corresponding sentence of the form of $\widetilde{\Delta}$ as $\widetilde{T}$.

Remark 3.7. A purely universal sentence $\forall u A_{0}(u)$ has the form of $\Delta$ and $\widetilde{\forall u A_{0}(u)}=\forall u A_{0}(u)$.

Definition $3.8([13,19])$. Let $\widehat{(\cdot)}^{1(1)}$ be a functional such that

$$
\widehat{f} n:= \begin{cases}f n & \text { if } f n \neq 0 \vee\left(\forall k, l(k * l=n \rightarrow f k=0) \wedge \forall i \leq l \operatorname{th} n\left((n)_{i} \leq 1\right)\right), \\ 1^{0} & \text { otherwise }\end{cases}
$$

and $(\cdot)_{(\cdot)}^{1(1)(1)}$ be a functional such that

$$
f_{g} n:= \begin{cases}f n & \text { if } f(g(l t h n))=0 \wedge l t h(g(l t h n))=l t h n, \\ 1^{0} & \text { otherwise }\end{cases}
$$

Then

$$
\mathrm{WKL}^{\prime}: \equiv \forall f^{1}, g^{1} \exists b \leq_{1} \lambda k \cdot 1 \forall x^{0}\left(\widehat{(\widehat{f})_{g}}(\bar{b} x)=_{0} 0\right)
$$

Note that WKL' has the form of $\Delta$. We recall that WKL is equivalent to WKL ${ }^{\prime}$ over $\widehat{W E-H A}{ }^{\omega} \uparrow([19$, Proposition 9.18.2]). See [16] and [19, Chapter 9, 10] for the detailed discussion on $\mathrm{WKL}^{\prime}$.

Definition $3.9([24,19,9]) . \Gamma_{2}\left(\subseteq \Gamma_{1}\right)$ is the syntactic class of formulas of $\mathscr{L}\left(\mathrm{E}-\mathrm{HA}{ }^{\omega}\right)$ (or $\mathscr{L}\left(\widehat{\mathrm{E}-\mathrm{HA}}^{\omega} \uparrow\right)$ in context) defined inductively as follows.

1. Prime formulas are in $\Gamma_{2}$.

2. If $A, B$ are in $\Gamma_{2}$, then $A \wedge B, A \vee B, \forall x A(x), \exists x A(x)$ are in $\Gamma_{2}$.

3. If $A$ is purely universal $\forall \underline{y}^{\rho} A_{0}(\underline{y})\left(A_{0}:\right.$ quantifier-free $)$ and $B \in \Gamma_{2}$, then $(\exists \underline{x} A \rightarrow B) \in \Gamma_{2}$. 
The next proposition is the extended variant of Theorem 5.6 in Hirst-Mummert [9].

Proposition 3.10. For a sentence $\mathrm{S}:=\forall x^{\rho}\left(A(x) \rightarrow \exists y^{\tau} B(x, y)\right)$ of $\mathscr{L}\left(\mathrm{WE}-\mathrm{HA}{ }^{\omega}\right)$ where $A(x)$ is purely universal and $B(x, y)$ is in $\Gamma_{2}$, if

$$
\mathrm{WE}-\mathrm{HA}{ }^{\omega}+\mathrm{AC}+\mathrm{IP}_{\forall}^{\omega}+\mathrm{M}^{\omega}+\Delta \vdash \mathrm{S},
$$

then

$$
\mathrm{WE}_{-\mathrm{PA}}{ }^{\omega}+\widetilde{\Delta} \vdash \mathrm{Uni}(\mathrm{S})
$$

In particular, if

$$
\mathrm{WE}-\mathrm{HA}{ }^{\omega}+\mathrm{AC}+\mathrm{IP}_{\forall}^{\omega}+\mathrm{M}^{\omega}+\mathrm{UWKL}+\mathrm{KL} \vdash \mathrm{S},
$$

then

$$
\text { WE-PA }{ }^{\omega}+\mathrm{UWKL} \vdash \mathrm{Uni}(\mathrm{S}) \text {. }
$$

This also holds for $\widehat{\mathrm{WE}-\mathrm{HA}}^{\omega} \uparrow$ and $\widehat{\mathrm{WE}}^{\mathrm{PA}}{ }^{\omega} \uparrow$ instead of $\mathrm{WE}^{-\mathrm{HA}}{ }^{\omega}$ and $\mathrm{WE}^{-\mathrm{PA}^{\omega}}$.

Proof. Since $A(x)$ is purely universal, $\forall x \exists y(A(x) \rightarrow B(x, y))$ is provable in $\mathrm{WE}^{-\mathrm{HA}}{ }^{\omega}+\mathrm{AC}+\mathrm{IP}_{\forall}^{\omega}+\mathrm{M}^{\omega}+\Delta$. Let denote $A(x) \rightarrow B(x, y)$ as $C(x, y)$ for convenience. Applying Theorem 9.1 from [19] ('soundness theorem for monotone functional interpretation') to the assumption, we have

$$
\mathrm{WE}-\mathrm{HA}^{\omega}+\widetilde{\Delta} \vdash \exists Y, \underline{U}\left(\forall x, \underline{v} C_{D}(x, Y x, \underline{U} x, \underline{v})\right)
$$

where $C^{D}=\exists \underline{u} \forall \underline{v} C_{D}(x, y, \underline{u}, \underline{v})$. Then, a fortiori, $\exists Y \forall x \exists \underline{u} \forall \underline{v} \underline{v} C_{D}(x, Y x, \underline{u}, \underline{v})$ follows. Since $C(x, Y x)$ is in $\Gamma_{2}$, applying [19, Lemma 8.11], we have

$$
\text { WE-HA }{ }^{\omega}+\widetilde{\Delta} \vdash \exists Y \forall x C(x, Y x) .
$$

Then, a fortiori, WE-PA ${ }^{\omega}+\widetilde{\Delta} \vdash \operatorname{Uni}(\mathrm{S})$. Taking $\Delta$ as $\widetilde{\mathrm{WKL}^{\prime}}$ (equivalent to UWKL, [19, Lemma 10.32]), in particular, $\widetilde{\Delta}=\Delta$. UWKL gives WKL and so, together with AC (see [19, Lemma 9.20]), KL.

The same proof works for the analogous result for $\widehat{\mathrm{WE}-\mathrm{HA}}^{\omega} \uparrow$ and $\widehat{\mathrm{WE}-\mathrm{PA}}^{\omega} \uparrow$.

Next, we refine the previous proposition to replace WE-HA ${ }^{\omega}$ by the full extensional system E-HA ${ }^{\omega}$. We use the techniques developed in [19, Section 10.4], which is a simplification of the treatment from [20].

Definition 3.11 (Translation for elimination of extensionality, [19]). Let $A$ be a formula of $\mathscr{L}\left(\mathrm{E}-\mathrm{HA}{ }^{\omega}\right)$ (or $\mathscr{L}\left(\widehat{\mathrm{E}-\mathrm{HA}}^{\omega} \uparrow\right)$ in context). $A_{e}$ is defined by induction on the logical structure of $A$ :

- $A_{e}: \equiv A$, if $A$ is a prime formula,

- $(A \square B)_{e}: \equiv\left(A_{e} \square B_{e}\right)$, where $\square \in\{\wedge, \vee, \rightarrow\}$,

- $\left(\exists x^{\rho} A\right)_{e}: \equiv \exists x^{\rho} A\left(x={ }_{\rho}^{e} x \wedge A_{e}\right)$,

- $\left(\forall x^{\rho} A\right)_{e}: \equiv \forall x^{\rho}\left(x={ }_{\rho}^{e} x \rightarrow A_{e}\right)$,

where the relation $x={ }_{\rho}^{e} y$ is defined by induction on $\rho$ as follows:

- $x==_{0}^{e} y: \equiv x={ }_{0} y$, 


$$
\text { - } x==_{\tau \rho}^{e} y: \equiv \forall u^{\rho}, v^{\rho}\left(u={ }_{\rho}^{e} v \rightarrow x u={ }_{\tau}^{e} x v \wedge x u={ }_{\tau}^{e} y v\right) \text {. }
$$

Remark 3.12. As observed by Ferreira [7], this definition can be shown to be equivalent to the simpler version where the first conjunct in $x={ }_{\tau \rho}^{e} y$ is dropped. However, we just follow [19, Section 10.4] in the present paper for convenience.

Lemma 3.13. For $x_{1}^{\rho}, x_{2}^{\rho}$ where $\rho=0 \rho_{k} \ldots \rho_{1}, x_{1}={ }_{\rho}^{e} x_{2} \leftrightarrow\left(x_{1}=\rho x_{2}\right)_{e} \wedge x_{2}={ }_{\rho}^{e} x_{2}$.

Proof. Note that by [19, Lemma 10.40.2],

$$
x_{1}={ }_{\rho}^{e} x_{2} \leftrightarrow \forall y_{1}^{\rho_{1}}, \tilde{y}_{1}^{\rho_{1}}, \ldots, y_{k}^{\rho_{k}}, \tilde{y}_{k}^{\rho_{k}}\left(\bigwedge_{i=1}^{k} y_{i}=\rho_{\rho_{i}}^{e} \tilde{y}_{i} \rightarrow x_{1} \underline{y}={ }_{0} x_{1} \underline{\tilde{y}} \wedge x_{1} \underline{y}={ }_{0} x_{2} \underline{\tilde{y}}\right) .
$$

On the other hand, $x_{1}=\rho x_{2}$ is the abbreviation of

$$
\forall y_{1}^{\rho_{1}}, \ldots, y_{k}^{\rho_{k}}\left(x_{1} y_{1}, \ldots, y_{k}={ }_{0} x_{2} y_{1} \ldots y_{k}\right) .
$$

One can verify the equivalence by a careful inspection with the use of [19, Lemma 10.37 and Lemma 10.39].

Lemma 3.14 (Elimination of extensionality). For every formula $A(\underline{a})$ of $\mathscr{L}\left(\mathrm{E}-\mathrm{HA}{ }^{\omega}\right)$ where a are all the free variables of $A$, if

$$
\mathrm{E}-\mathrm{HA}^{\omega}+\mathrm{AC} !^{1}+\mathrm{AC}^{0} \vdash A(\underline{a})
$$

then

$$
\mathrm{WE}-\mathrm{HA}^{\omega}+\mathrm{AC} \vdash \underline{a}={ }^{e} \underline{a} \rightarrow A(\underline{a}) .
$$

This also holds for $\widehat{\mathrm{E}-\mathrm{HA}}^{\omega} \uparrow$ and $\widehat{\mathrm{WE}-\mathrm{HA}}^{\omega} \uparrow$ instead of $\mathrm{E}-\mathrm{HA}^{\omega}$ and $\mathrm{WE}-\mathrm{HA}^{\omega}$.

Proof. By induction on the derivation. In fact, the proof is the same as for [19, Proposition 10.45] (see also [19, Remark 10.46.2]) except the interpretation of $A C !^{1}$ and $\mathrm{AC}^{0}$. We only discuss their interpretation in WE-HA ${ }^{\omega}+$ AC (see [20] for more details).

Since $x={ }_{\rho}^{e} x$ holds for $x^{\rho}$ such that $\operatorname{deg}(\rho) \leq 1([19, \operatorname{Lemma} 10.41]),\left(\mathrm{AC} !^{\rho, \tau}\right)_{e}$ where $\operatorname{deg}(\rho) \leq 1$ is equivalent to the following formula (with implicit parameters $\underline{p}$ );

$$
\begin{gathered}
\forall x^{\rho} \exists y^{\tau}\left(y={ }_{\tau}^{e} y \wedge A(x, y)_{e} \wedge \forall z^{\tau}\left(z={ }_{\tau}^{e} z \wedge A(x, z)_{e} \rightarrow\left(y={ }_{\tau} z\right)_{e}\right)\right) \\
\rightarrow \quad \exists Y^{\tau \rho}\left(Y={ }_{\tau \rho}^{e} Y \wedge \forall x^{\rho} A(x, Y x)_{e}\right) .
\end{gathered}
$$

Assume (1). Then we have

$$
\exists Y^{\tau \rho} \forall x\left(Y x={ }_{\tau}^{e} Y x \wedge A(x, Y x)_{e} \wedge \forall z^{\tau}\left(z={ }_{\tau}^{e} z \wedge\left(A(x, z)_{e} \rightarrow\left(Y x={ }_{\tau} z\right)_{e}\right)\right)\right.
$$

by $\mathrm{AC}^{\rho, \tau}$. What we have to show is only that such a $Y$ is extensional, i.e. $\forall x_{1}^{\rho}, x_{2}^{\rho}\left(x_{1}={ }_{\rho}^{e} x_{2} \rightarrow Y x_{1}={ }_{\tau}^{e} Y x_{2}\right)$. Note that

$$
Y x_{1}={ }_{\tau}^{e} Y x_{2} \equiv \forall v_{1}^{\tau_{1}}, v_{2}^{\tau_{1}}\left(v_{1}={ }_{\tau_{1}}^{e} v_{2} \rightarrow Y x_{1} v_{1}={ }_{\tau_{2}}^{e} Y x_{1} v_{2} \wedge Y x_{1} v_{1}={ }_{\tau_{2}}^{e} Y x_{2} v_{2}\right)
$$

where $\tau=\tau_{2} \tau_{1}$. On one hand, $Y x_{1} v_{1}={ }^{e} Y x_{1} v_{2}$ is a direct consequence of $Y x_{1}={ }_{\tau}^{e} Y x_{1}$. On the other hand, $Y x_{1} v_{1}={ }^{e} Y x_{2} v_{2}$ follows form $Y x_{2}={ }^{e} Y x_{2}$ and $Y x_{2}={ }^{e} Y x_{2} \wedge A\left(x_{1}, Y x_{2}\right)_{e} \rightarrow\left(Y x_{1}={ }_{\tau} Y x_{2}\right)_{e}$ via Lemma 3.13, since 
$A\left(x_{1}, Y x_{2}\right)_{e}$ follows from $x_{1}={ }^{e} x_{2}$ and $A\left(x_{2}, Y x_{2}\right)_{e}$ (note that each term $t[\underline{p}]$ occurring in $A(x, y)$ is extensional under the assumption $p={ }^{e} p[19$, Lemma 10.42]).

Next we turn to the interpretation of $\mathrm{AC}^{0}$. For any type $\tau,\left(\mathrm{AC}^{0, \tau}\right)_{e}$ is equivalent to $\forall x^{0} \exists y^{\tau}\left(y={ }_{\tau}^{e} y \wedge A(x, y)_{e}\right)$ $\rightarrow \exists Y^{\tau(0)}\left(Y={ }_{\tau}^{e} Y \wedge \forall x\left(A(x, Y x)_{e}\right)\right)$. This is derived from $\mathrm{AC}^{0, \tau}$ applied to $\forall x^{0} \exists y^{\tau}\left(y={ }_{\tau}^{e} y \wedge A(x, y)_{e}\right)$ with the use of the fact that the full extensionality for equality of type 0 holds in WE-HA ${ }^{\omega}$ ([19, Remark 3.13.2]).

The same proof works for the analogous result for $\widehat{\mathrm{E}-\mathrm{HA}} \omega^{\omega} \uparrow$ and $\widehat{\mathrm{WE}-\mathrm{HA}}{ }^{\omega} \uparrow$.

We are now in the position to refine Proposition 3.10. Taking applications into account, we state the refined one in the form of being able to obtain the best possible unprovability results from the results in reverse mathematics.

Proposition 3.15. Let $\Delta_{1}^{*}$ be the class of sentences $\forall a^{\rho} \exists b \leq_{1} r a \forall c^{\tau} B_{0}(a, b, c)$ (where $r$ is a closed term and $\rho, \tau$ are arbitrary types) such that $\widetilde{\Delta_{1}^{*}}$ is provable in $\mathrm{WE}-\mathrm{PA}{ }^{\omega}+\mathrm{QF}-\mathrm{AC}+\mathrm{UWKL}$.

For every statement $\mathrm{S}:=\forall x^{\rho}\left(A(x) \rightarrow \exists y^{\tau} B(x, y)\right)$ of $\mathscr{L}\left(\mathrm{E}^{-\mathrm{HA}}{ }^{\omega}\right)$ where $A(x)$ is purely universal, $B(x, y)$ is in $\Gamma_{2}$ and the types of all variables quantified in $S$ by positively occurring $\forall$ or negatively occurring $\exists$ are not greater than 1 (in particular, $\rho \leq 1$ ), if

$$
\mathrm{E}-\mathrm{HA}{ }^{\omega}+\mathrm{AC} !^{1}+\mathrm{AC}^{0}+\mathrm{IP}_{\forall}^{\leq 1, \leq 1}+\mathrm{M}^{\leq 1}+\mathrm{KL}+\Delta_{1}^{*} \vdash \mathrm{S},
$$

then

$$
\mathrm{WE}^{-\mathrm{PA}^{\omega}}+\mathrm{QF}-\mathrm{AC}+\mathrm{UWKL} \vdash \mathrm{Uni}(\mathrm{S}) .
$$

This also holds for $\widehat{\mathrm{E}-\mathrm{HA}}^{\omega} \uparrow$ and $\widehat{\mathrm{WE}-\mathrm{PA}}^{\omega} \uparrow$ instead of $\mathrm{E}-\mathrm{HA}^{\omega}$ and $\mathrm{WE}-\mathrm{PA}^{\omega}$.

Proof. We may assume that $\mathrm{IP}_{\forall}^{\leq 1, \leq 1}, \mathrm{M}^{\leq 1}$ and $\Delta_{1}^{*}$ are finite, so can form the conjunction of their elements. We also denote them as $\operatorname{IP}_{\forall}^{\leq 1, \leq 1}, \mathbf{M}^{\leq 1}$ and $\Delta_{1}^{*}$ for readability. Take the universal closures of $\operatorname{IP}_{\forall}^{\leq 1, \leq 1}$ and $\mathbf{M}^{\leq 1}$, and denote them as $\overline{\mathrm{IP}_{\forall}^{\leq 1, \leq 1}}$ and $\overline{\mathrm{M} \leq 1}$ respectively (note that $\mathrm{IP}_{\forall}^{\leq 1, \leq 1}$ and $\mathrm{M}^{\leq 1}$ may have parameters of arbitrary types). Then we have

$$
\mathrm{E}-\mathrm{HA}{ }^{\omega}+\mathrm{AC} !^{1}+\mathrm{AC}^{0} \vdash\left(\overline{\mathrm{IP}_{\forall}^{\leq 1, \leq 1}} \wedge \overline{\mathrm{M} \leq 1} \wedge \mathrm{KL} \wedge \Delta_{1}^{*}\right) \rightarrow \mathrm{S}
$$

by the deduction theorem. Applying Lemma 3.14, we have

$$
\mathrm{WE}-\mathrm{HA} \mathrm{A}^{\omega}+\mathrm{AC} \vdash\left(\left(\overline{\mathrm{IP}_{\forall}^{\leq 1, \leq 1}}\right)_{e} \wedge(\overline{\mathrm{M} \leq 1})_{e} \wedge(\mathrm{KL})_{e} \wedge\left(\Delta_{1}^{*}\right)_{e}\right) \rightarrow(\mathrm{S})_{e},
$$

since $\mathrm{S}$ has no parameter as well as $\overline{\mathrm{IP}_{\forall}^{\leq 1, \leq 1}}, \overline{\mathrm{M} \leq 1}, \mathrm{KL}$ and $\Delta_{1}^{*}$. By a careful inspection with the use of [19, Lemma 10.41] and the restriction of the types of variables in $\operatorname{IP}_{\forall}^{\leq 1, \leq 1}, M^{\leq 1}$ and $S$, one can intuitionistically show that $\overline{\mathrm{IP}_{\forall}^{\leq 1, \leq 1}} \rightarrow\left(\overline{\mathrm{IP}_{\forall}^{\leq 1, \leq 1}}\right)_{e}, \overline{\mathrm{M} \leq 1} \rightarrow(\overline{\mathrm{M} \leq 1})_{e}, \mathrm{KL} \leftrightarrow(\mathrm{KL})_{e}, \Delta_{1}^{*} \rightarrow\left(\Delta_{1}^{*}\right)_{e}$ and $(\mathrm{S})_{e} \rightarrow \mathrm{S}$. Therefore we have

$$
\mathrm{WE}-\mathrm{HA}^{\omega}+\mathrm{AC}+\overline{\mathrm{IP}_{\forall}^{\leq 1, \leq 1}}+\overline{\mathrm{M} \leq 1}+\mathrm{KL}+\Delta_{1}^{*} \vdash \mathrm{S} .
$$

Then WE-PA ${ }^{\omega}+$ QF-AC + UWKL $\vdash$ Uni(S) follows from Proposition 3.10.

The same proof works for the analogous result for $\widehat{\mathrm{E}-\mathrm{HA}}^{\omega} \uparrow$ and $\widehat{\mathrm{WE}-\mathrm{PA}}^{\omega} \uparrow$. 
Remark 3.16. 1. Since $\mathrm{QF}-\mathrm{AC}^{1,0} \leftrightarrow \mathrm{QF}-\mathrm{AC} !^{1,0}$ over $\widehat{\mathrm{WE}-\mathrm{HA}}^{\omega} \uparrow$, the intuitionistic version $\mathrm{iWKL}_{0}^{\omega}:=$ $\widehat{\mathrm{E}-\mathrm{HA}}^{\omega} \uparrow+\mathrm{QF}-\mathrm{AC}^{1,0}+\mathrm{WKL}$ of $\mathrm{WKL}_{0}^{\omega}$ in $[18]$ is a subsystem of $\widehat{\mathrm{E}-\mathrm{HA}}^{\omega} \uparrow+\mathrm{AC}^{1}+\mathrm{AC}^{0}+\mathrm{IP}_{\forall}^{\leq 1, \leq 1}+$ $\mathrm{M}^{\leq 1}+\mathrm{KL}$. That is to say, the contrapositive of the previous proposition yields the unprovability of $S$ in $\mathrm{iWKL}_{0}^{\omega}$ or $\mathrm{iWKL}^{\omega}:=\mathrm{E}-\mathrm{HA}{ }^{\omega}+\mathrm{QF}^{-A C^{1,0}}+\mathrm{WKL}$.

2. All sentences $\forall x^{\rho} \exists y^{\tau} A_{0}(x, y)$ provable in $\mathrm{RCA}^{\omega}$, where the degree of the type $\rho$ is not greater than 1 , the type $\tau$ is arbitrary and $A_{0}$ is quantifier-free, are included in $\mathrm{E}-\mathrm{HA}{ }^{\omega}+\mathrm{AC} !^{1}+\mathrm{AC}^{0}+\mathrm{IP}_{\forall}^{\leq 1, \leq 1}+\mathrm{M}^{\leq 1}+$ $\mathrm{KL}+\Delta_{1}^{*}$ as in Remark 3.5.

3. The type restriction for $\mathrm{S}$ in the previous proposition still covers $\Pi_{2}^{1}$-sentences treated in reverse mathematics.

Corollary 3.17. Let $\Delta_{1}^{*}$ be the same as in Proposition 3.15. For every statement $\mathrm{S}:=\forall x^{1}\left(A(x) \rightarrow \exists y^{1} B(x, y)\right)$ of $\mathscr{L}\left(\mathrm{E}-\mathrm{HA}^{2}\right)$ where $A(x)$ is purely universal and $B(x, y)$ is in $\Gamma_{2}$, if

$$
\mathrm{E}-\mathrm{HA}{ }^{\omega}+\mathrm{AC} !^{1}+\mathrm{AC}^{0}+\mathrm{IP}_{\forall}^{\leq 1, \leq 1}+\mathrm{M}^{\leq 1}+\mathrm{KL}+\Delta_{1}^{*} \vdash \mathrm{S},
$$

then

$$
\mathrm{WE}_{-\mathrm{PA}}{ }^{\omega}+\mathrm{QF}-\mathrm{AC}+\mathrm{UWKL} \vdash \mathrm{Seq}(\mathrm{S})\left(:=\forall x^{1(0)}\left(\forall n^{0} A(x n) \rightarrow \exists y^{1(0)} \forall n B(x n, y n)\right)\right) .
$$

Proof. Immediate consequence of Proposition 3.15 since Uni(S) derives Seq(S).

Remark 3.18. The above corollary is applicable to some results in sequential reverse mathematics. Suppose the sequential version of a statement $\mathrm{S}$ where $A(x)$ is purely universal and $B(x, y)$ is in $\Gamma_{2}$ derives ACA over $\mathrm{RCA}^{\omega}$. Then we have WE-PA ${ }^{\omega}+\mathrm{QF}_{-\mathrm{AC}}{ }^{1,0}+\mathrm{Seq}(\mathrm{S}) \vdash \mathrm{ACA}$ by elimination of extensionality [19, Proposition 10.45]. It is known that $\mathrm{WE}_{\mathrm{PA}}{ }^{\omega}+\mathrm{ACA}$ proves the totality of the $\alpha<\varepsilon_{\varepsilon_{0}}$-recursive functions ([6]). On the other hand, the provably recursive functions of $\mathrm{WE}-\mathrm{PA}{ }^{\omega}+\mathrm{QF}-\mathrm{AC}+\mathrm{UWKL}$ are the $\alpha<\varepsilon_{0}$-recursive functions (see [19, Corollary 33] and note that Gödel primitive recursive functionals of type degree 1 coincide with provably recursive functions of $\mathrm{PA})$. Therefore $\mathrm{Seq}(\mathrm{S})$ is not provable in $\mathrm{WE}-\mathrm{PA}{ }^{\omega}+\mathrm{QF}-\mathrm{AC}+\mathrm{UWKL}$. By applying the previous corollary, we have the unprovability of $\mathrm{S}$ in $\mathrm{E}-\mathrm{HA}{ }^{\omega}+\mathrm{AC} !^{1}+\mathrm{AC}^{0}+\mathrm{IP}_{\forall}^{\leq 1, \leq 1}+\mathrm{M}^{\leq 1}+\mathrm{KL}+\Delta_{1}^{*}$. That is to say, one can think of Corollary 3.17 (essentially Proposition 3.15) as a kind of higher-order uniformization theorem for RCA + WKL ([*] in table 1). As we see in the subsequent sections, nevertheless, one can obtain the much stronger semi-intuitionistic unprovability for such statements by investigating the strength of uniform versions over a weakly extensional classical system like WRCA ${ }^{\omega}$.

\section{Main results}

The model $\mathscr{M}^{\omega}$ of all strongly majorizable functionals, which was first introduced in Bezem [3], is a crucial tool for our result.

Definition $4.1([19,12,14])$. The type structure $\mathscr{M}^{\omega}$ of all hereditarily strongly majorizable set-theoretic functionals of finite type is defined as

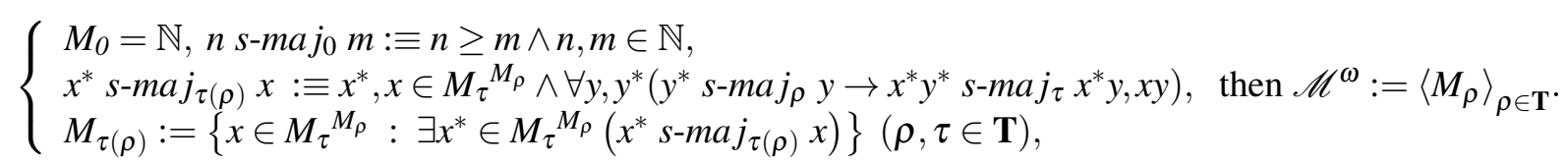

(Here $M_{\tau}{ }^{M_{\rho}}$ denotes the set of all total set-theoretic functions from $M_{\rho}$ to $M_{\tau}$.) 
In addition, we recall the following definitions (see [19, Chapter 12] for the detailed discussion on $\mathrm{F}^{(-)}$and $\Sigma_{1}^{0}$-UB ${ }^{(-)}$and see e.g. $[2,10,11,19]$ for general information on $(\mathrm{BI})$ and $\left.(\mathrm{BR})\right)$ :

- For $z^{\rho(0)},(\overline{z, n})\left(k^{0}\right):=\rho\left\{\begin{array}{ll}z k & \text { if } k<_{0} n, \\ 0^{\rho} & \text { otherwise, }\end{array}\right.$ where $0^{\rho}$ is constant- 0 functional of type $\rho$.

- $\mathrm{F}: \forall \Phi^{2(0)}, y^{1(0)}, \exists y_{0} \leq_{1(0)} y \forall k^{0}, z \leq_{1} y k\left(\Phi k z \leq_{0} \Phi k\left(y_{0} k\right)\right)$.

$\mathrm{F}^{-}: \forall \Phi^{2(0)}, y^{1(0)}, \exists y_{0} \leq_{1(0)} y \forall k^{0}, z^{1}, n^{0}\left(\bigwedge_{i<{ }_{0} n} z i \leq_{0} y k i \rightarrow \Phi k(\overline{z, n}) \leq_{0} \Phi k\left(y_{0} k\right)\right)$.

- $\Sigma_{1}^{0}$-UB : $\forall y^{1(0)}\left(\forall k^{0} \forall x \leq_{1} y k \exists z^{0} A(x, y, k, z) \rightarrow \exists \chi^{1} \forall k^{0} \forall x \leq_{1} y k \exists z \leq_{0} \chi k A(x, y, k, z)\right)$,

$\Sigma_{1}^{0} \mathrm{UB}^{-}: \forall y^{1(0)}\left(\forall k^{0} \forall x \leq_{1} y k \exists z^{0} A(x, y, k, z) \rightarrow \exists \chi^{1} \forall k^{0}, x^{1}, n^{0}\left(\bigwedge_{i<n}\left(x i \leq_{0} y k i\right) \rightarrow \exists z \leq_{0} \chi k A((\overline{x, n}), y, k, z)\right)\right)$, where $A \equiv \exists \underline{l} A_{0}(\underline{l})$ and $\underline{l}$ is a tuple of variables of type 0 and $A_{0}$ is a quantifier-free formula which may contain parameters of arbitrary types.

- KL (König's lemma): $\forall f^{1}\left(\tilde{T}^{\infty}(f) \rightarrow \exists b^{1} \forall x^{0}(f(\bar{b} x)=0)\right)$, where $\tilde{T}^{\infty}(f)$ expresses that $f$ represents a finitely branching infinite tree.

- $\mathrm{DC}^{\rho}: \forall x^{0}, y^{\rho} \exists z^{\rho} A(x, y, z) \rightarrow \exists f^{\rho(0)} \forall x^{0} A(x, f(x), f(S(x)))$.

DC (dependent choice) $:=\bigcup_{\rho \in \mathbf{T}}\left\{\mathrm{DC}^{\rho}\right\}$.

- $\left(\mathrm{BI}_{\rho}\right): \forall x^{\rho 0}, n^{0}\left(\begin{array}{l}\exists k^{0} P(\overline{x, k} ; k) \wedge \\ P(\overline{x, n} ; n) \rightarrow P(\overline{x, n+1} ; n+1) \wedge \\ P(\overline{x, n} ; n)=0 \rightarrow Q(\overline{x, n} ; n) \wedge \\ \forall u^{\rho} Q(\overline{x, n} * u ; n+1) \rightarrow Q(\overline{x, n} ; n)\end{array}\right) \rightarrow Q\left(0^{\rho 0} ; 0^{0}\right)$,

where $P, Q$ are arbitrary formulas and

$$
(\overline{x, n} * u) k=\rho \begin{cases}x k & \text { if } k<n \\ u & \text { if } k=n \\ 0^{\rho} & \text { otherwise }\end{cases}
$$

$\mathrm{BI}$ (bar induction) $:=\bigcup_{\rho \in \mathbf{T}}\left\{\mathrm{BI}_{\rho}\right\}$.

$\mathrm{BI}^{\leq 1}:=\bigcup_{\rho \in \mathbf{T}, \operatorname{deg}(\rho) \leq 1}\left\{\mathrm{BI}_{\rho}\right\}$.

- $\mathrm{BR}_{\rho, \tau}:\left\{\begin{array}{l}y(\overline{x, n})<_{0} n \rightarrow B_{\rho, \tau} y z u n x=\tau z n(\overline{x, n}) \\ y(\overline{x, n}) \geq_{0} n \rightarrow B_{\rho, \tau} y z u n x=\tau u\left(\lambda D^{\rho} . B_{\rho, \tau} y z u n x(n+1)(\overline{x, n} * D)\right) n(\overline{x, n})\end{array}\right.$

for all $x^{\rho(0)}$ and $n^{0}$.

BR (bar recursion) $:=\bigcup_{\rho, \tau \in \mathbf{T}}\left\{\mathrm{BR}_{\rho, \tau}\right\}$.

If the system in question has BR, we implicitly assume that new constants $B_{\rho, \tau}$ for bar recursion are added. The important thing for our analysis is that BR is a purely universal axiom scheme.

Remark 4.2. Bar induction in all finite types is a generalization of Brouwer's 'bar theorem' considered first by Spector [23]. Spector also defined the new concept of bar recursion. The precise formulations of (BI) and (BR) used above are taken from [20] (see also [10] and Section 11.1 from [19]). 
The following is our main theorem.

Theorem 4.3. Let $\Delta^{\mathscr{M}^{\omega}}$ be a set of sentences of the form $\Delta$ which are true in $\mathscr{M}^{\omega}$. For every statement $\forall x^{\rho} \exists y^{\tau} A(x, y)$ of $\mathscr{L}\left(\mathrm{WE}^{\mathrm{H}} \mathrm{HA}{ }^{\omega}\right)$ in $\Gamma_{2}$, if

$$
\text { WE-PA }{ }^{\omega}+\mathrm{QF}-\mathrm{AC}+\mathrm{DC}+\Delta^{\mathscr{M}^{\omega}}+\exists Y^{\tau(\rho)} \forall x^{\rho} A(x, Y x) \vdash\left(\exists^{2}\right),
$$

then

$$
\mathrm{WE}-\mathrm{HA}^{\omega}+\mathrm{AC}+\mathrm{IP}_{\forall}^{\omega}+\mathrm{M}^{\omega}+\mathrm{BR}+\mathrm{F}^{-}+\Delta^{\mathscr{M}^{\omega}} \nvdash \forall x \exists y A(x, y) .
$$

In particular, if

$$
\mathrm{WE}-\mathrm{PA}{ }^{\omega}+\mathrm{QF}-\mathrm{AC}+\mathrm{DC}+\mathrm{UWKL}+\exists Y^{\tau(\rho)} \forall x^{\rho} A(x, Y x) \vdash\left(\exists^{2}\right),
$$

then

$$
\mathrm{WE}-\mathrm{HA}^{\omega}+\mathrm{AC}+\mathrm{IP}_{\forall}^{\omega}+\mathrm{M}^{\omega}+\mathrm{UWKL}+\mathrm{KL}+\Sigma_{1}^{0}-\mathrm{UB}^{-}+\mathrm{BI} \nvdash \forall x \exists y A(x, y) .
$$

We should note that $\mathrm{F}^{-}$and $\Sigma_{1}^{0}-\mathrm{UB}^{-}$are involved primarily for technical reasons (see also Remark 4.11). The proof is based on the Dialectica interpretation without extracting terms/bounds (Lemma 4.9) and negative translation along with the model $\mathscr{M}^{\omega}$ of all strongly majorizable functionals.

Remark 4.4. To obtain the conclusion of Theorem 4.3 it is not enough to check that over WE-PA ${ }^{\omega}+\mathrm{QF}-\mathrm{AC}$ the uniform version (or even just the sequential version) of $\forall x^{1} \exists y^{1} A$ implies ACA: define

$$
A:=\forall f^{1(0)}, \varphi^{2}, \psi^{2} \exists g^{1}(f(\varphi(g), \psi(g))=0 \rightarrow f(\varphi(g), g(\psi(g)))=0) \in \Gamma_{2}
$$

and add 'dummy variables' to get $\forall x^{1} \exists y^{1} A$. Then this statement coincides with its sequential (as well as its full uniform) version and implies (using classical logic and $\mathrm{QF}-\mathrm{AC}$ ) ACA since it implies

$$
\forall f^{1(0)} \exists g^{1} \forall n^{0}, k^{0}(f(n, k)=0 \rightarrow f(n, g(n))=0) .
$$

But A is provable in $\mathrm{WE}-\mathrm{HA}{ }^{\omega}+\mathrm{BR}$ since it has a functional interpretation in this theory.

We first show some lemmas used in the proof. Since they are not difficult, we only sketch the proofs.

Definition 4.5 (Kuroda's negative translation, [19]). Let $A$ be a formula of $\mathscr{L}\left(\mathrm{E}^{\mathrm{P} A}{ }^{\omega}\right)$ (or $\mathscr{L}\left(\widehat{\mathrm{E}-\mathrm{PA}}^{\omega} \uparrow\right)$ in context). $A^{\prime}$ is defined as $A^{\prime}: \equiv \neg \neg A^{*}$, where $A^{*}$ is defined by induction on the logical structure of $A$ :

- $A^{*}: \equiv A$, if $A$ is a prime formula,

- $(A \square B)^{*}: \equiv\left(A^{*} \square B^{*}\right)$, where $\square \in\{\wedge, \vee, \rightarrow\}$,

- $\left(\exists x^{\rho} A\right)^{*}: \equiv \exists x^{\rho} A^{*}$,

- $\left(\forall x^{\rho} A\right)^{*}: \equiv \forall x^{\rho} \neg \neg A^{*}$.

See [19, Section 10.1] for the detailed discussion on negative translation. 
Lemma 4.6. For every formula $A$ and $B$ of $\mathscr{L}\left(\mathrm{WE}^{-\mathrm{PA}^{\omega}}\right)$, if

$$
\mathrm{WE}_{-\mathrm{PA}}{ }_{-}^{+} \mathrm{QF}_{-} \mathrm{AC}_{-}^{+} \Delta_{-}^{+} A \vdash B
$$

then

$$
\mathrm{WE}-\mathrm{HA}{ }_{-}^{\omega+}\left\{\mathrm{QF}-\mathrm{AC}+\mathrm{M}^{\omega}\right\}{ }_{-}^{+} \Delta_{-}^{+} A^{\prime} \vdash B^{\prime},
$$

where $\mathscr{T}_{-}^{+} \mathrm{T}$ indicates that we consider both case: $\mathscr{T}$ and $\mathscr{T}+\mathrm{T}$. This also holds for $\widehat{\mathrm{WE}^{-\mathrm{PA}}}{ }^{\omega} \uparrow$ and $\widehat{\mathrm{WE}-\mathrm{HA}}{ }^{\omega} \uparrow$ instead of WE-PA ${ }^{\omega}$ and $\mathrm{WE}-\mathrm{HA}^{\omega}$.

Proof. Induction on the length of the derivation as for [19, Proposition 10.3] (see also [19, Proposition 10.6, Proposition 10.19]). Note that $\mathrm{M}^{\omega}$ is only used to verify the negative translation of QF-AC.

Remark 4.7. By [10] (and [20]), classical analysis $\mathrm{E}-\mathrm{PA}^{\omega}+\mathrm{DC}$ embeds into $\mathrm{WE}-\mathrm{HA}{ }^{\omega}+\mathrm{BI}+\mathrm{AC}+\mathrm{IP}_{\forall}^{\omega}+\mathrm{M}^{\omega}$ by (elimination of extensionality and) negative translation.

Lemma 4.8. $\widehat{\mathrm{WE}-\mathrm{H} A}{ }^{\omega} \uparrow+\mathrm{M}^{0} \vdash\left(\exists^{2}\right)^{\prime} \rightarrow \neg \neg\left(\exists^{2}\right)$.

Proof. Reasoning in $\widehat{\mathrm{WE}_{\mathrm{HA}}}{ }^{\omega} \uparrow+\mathrm{M}^{0}$,

$$
\begin{aligned}
\left(\exists^{2}\right)^{*} & =\exists E \forall f \neg \neg(E f=0 \leftrightarrow \exists x(f x=0)) \\
& \rightarrow \exists E \forall f([E f=0 \rightarrow \neg \neg \exists x(f x=0)] \wedge[\exists x(f x=0) \rightarrow \neg \neg E f=0]) \\
& \left.\rightarrow \exists E \forall f([E f=0 \rightarrow \exists x(f x=0)] \wedge[\exists x(f x=0) \rightarrow E f=0]) \quad \text { (using } \mathbf{M}^{0}\right) \\
& =\left(\exists^{2}\right) .
\end{aligned}
$$

Therefore $\left(\exists^{2}\right)^{\prime}=\neg \neg\left(\exists^{2}\right)^{*} \rightarrow \neg \neg\left(\exists^{2}\right)$.

The next lemma is just the simple variant of Theorem 11.9 in Kohlenbach [19], where we do not insist on the existence of witnessing terms for $(\forall \underline{a} A(\underline{a}))^{D}$ (nor uniform bounds) and so can add axioms $\Delta$ (without having to formalize the majorizability proof of $\mathrm{BR}$ as in the monotone functional interpretation).

Lemma 4.9 (Soundness of the Dialectica interpretation without extracting terms/bounds). Let $A(\underline{a})$ be a formula of $\mathscr{L}\left(\mathrm{WE}^{\mathrm{H}} \mathrm{A}^{\omega}\right)$ containing only a free. Then if

$$
\mathrm{WE}-\mathrm{HA}^{\omega}+\mathrm{AC}+\mathrm{IP}_{\forall}^{\omega}+\mathrm{M}^{\omega}+\Delta\left(+\mathrm{BR}+\mathrm{DC}^{\prime}\right) \vdash A(\underline{a}),
$$

then

$$
\mathrm{WE}-\mathrm{HA}^{\omega}+\widetilde{\Delta}(+\mathrm{BR}) \vdash(\forall \underline{a} A(\underline{a}))^{D},
$$

where $(\forall \underline{a} A(\underline{a}))^{D}$ is the Dialectica interpretation [19, Definition 8.1] of $\forall \underline{a} A(\underline{a})$ and $\mathrm{DC}^{\prime}$ denotes the negative translation of DC.

Proof. As in the proof of the soundness theorem for the Dialectica interpretation extracting terms ([19, Theorem 8.6]), we proceed by induction on the length of the derivation. Note that our interpretation of $A(\underline{a})$ is not the Dialectica interpretation of $A(\underline{a})$ but that of the universal closure of $A(\underline{a})$. For the axioms of WE-HA ${ }^{\omega}, \mathrm{AC}, \mathrm{IP}_{\forall}^{\omega}$ and $\mathrm{M}^{\omega}$, each induction step immediately follows from the corresponding step in the proof of [19, Theorem 8.6]. For the rules of $W E-H A{ }^{\omega}$, on the other hand, each induction step follows by imitating the construction to the witness term from the given terms in the corresponding step for [19, Theorem 8.6] (with the use of [19, Lemma 3.15] and [19, Remark 3.13.2]). In addition, the interpretation of $\Delta$ is $\widetilde{\Delta}$ (here we use that $\Delta$ only contains 'sentences'). DC' is interpreted by BR as in the proof of [19, Theorem 11.9]. 
The next lemma states that $\Delta^{\mathscr{M}^{\omega}}$ is closed under ${ }^{\sim}$ transformation. Note that for $\mathrm{T}$ of the form $\Delta, \widetilde{\mathrm{T}}$ also has the form of $\Delta$.

Lemma 4.10. If a sentence $\mathrm{T}$ of $\mathscr{L}\left(\mathrm{E}-\mathrm{HA}^{\omega}\right)\left(\right.$ or $\mathscr{L}\left(\widehat{\mathrm{E}-\mathrm{HA}}^{\omega} \uparrow\right)$ in context $)$ has the form of $\Delta$ and $\mathscr{M} \models \mathrm{T}$, then $\mathscr{M} \models \widetilde{\mathrm{T}}$ holds.

Proof. Note that $\mathrm{T} \rightarrow \widetilde{\mathrm{T}}$ is derived from $\mathrm{b}-\mathrm{AC}$ where $\mathrm{b}-\mathrm{AC}: \equiv \bigcup_{\rho, \tau}\left\{\mathrm{b}-\mathrm{AC}{ }^{\rho, \tau}\right\}$ with

$$
\text { b-AC }{ }^{\rho, \tau}: \equiv \forall Z^{\tau \rho}\left(\forall x^{\tau} \exists y \leq_{\rho} Z x A(x, y, Z) \rightarrow \exists Y \leq_{\rho \tau} Z \forall x A(x, Y z, Z)\right) .
$$

Since $\mathscr{M}^{\omega}$ models E-PA ${ }^{\omega}+\mathrm{b}-\mathrm{AC}([12$, Application 3.12.1]), $\mathscr{M} \models \widetilde{\mathrm{T}}$ follows from $\mathscr{M} \models \mathrm{T}$.

We are now prepared to show Theorem 4.3.

Proof of Theorem 4.3. Suppose that $(i)$ holds but (ii) does not hold for some $\forall x \exists y A(x, y) \in \Gamma_{2}$. Note that $\mathrm{F}^{-}$ has the form $\Delta$. Applying Lemma 4.9 to (the negation of) (ii), we have

$$
\mathrm{WE}-\mathrm{HA}^{\omega}+\mathrm{BR}+\widetilde{\mathrm{F}^{-}}+\widetilde{\Delta^{\mathscr{M}^{\omega}}} \vdash \exists Y \forall x \exists \underline{u} \forall \underline{v} A_{D}(x, Y x, \underline{u}, \underline{v})
$$

where $A^{D}=\exists \underline{u} \forall \underline{v} A_{D}(x, y, \underline{u}, \underline{v})$. Since $A(x, Y x)$ is in $\Gamma_{2}$, applying [19, Lemma 8.11],

$$
\mathrm{WE}-\mathrm{HA}{ }^{\omega}+\mathrm{BR}+\widetilde{\mathrm{F}^{-}}+\widetilde{\Delta^{\mathscr{M}^{\omega}}} \vdash \exists Y \forall x A(x, Y x)
$$

follows. Since $\mathrm{BR}^{\prime},\left(\widetilde{\mathrm{F}^{-}}\right)^{\prime}$ and $\left(\widetilde{\Delta^{\mathscr{M}^{\omega}}}\right)^{\prime}$ are derived from $\mathrm{BR}, \widetilde{\mathrm{F}^{-}}$and $\widetilde{\Delta^{\mathscr{M}}}$ respectively, we have

$$
\mathrm{WE}-\mathrm{HA}{ }^{\omega}+\mathrm{BR}+\widetilde{\mathrm{F}^{-}}+\widetilde{\Delta^{\mathscr{M}^{\omega}}} \vdash(\exists Y \forall x A(x, Y x))^{\prime}
$$

from Lemma 4.6. On the other hand, Lemma 4.6 applied to $(i)$ yields

$$
\mathrm{WE}-\mathrm{HA}{ }^{\omega}+\mathrm{QF}-\mathrm{AC}+\mathrm{DC}^{\prime}+\Delta^{\mathscr{M}^{\omega}}+\mathrm{M}^{\omega}+(\exists Y \forall x A(x, Y x))^{\prime} \vdash\left(\exists^{2}\right)^{\prime} .
$$

Combining the proofs $\left(i^{\prime}\right)$ and $(i i)^{\prime}$ (note that $\Delta^{\mathscr{M}^{\omega}}$ is derived from $\widetilde{\Delta^{\mathscr{M}^{\omega}}}$ ), we have a proof

$$
\mathrm{WE}-\mathrm{HA}{ }^{\omega}+\mathrm{QF}-\mathrm{AC}+\mathrm{DC}^{\prime}+\mathrm{M}^{\omega}+\mathrm{BR}+\widetilde{\mathrm{F}^{-}}+\widetilde{\Delta^{\mathscr{M}^{\omega}}} \vdash\left(\exists^{2}\right)^{\prime}
$$

Now we show that this leads to a contradiction. Since we have $\left(\mu^{2}\right)$ intuitionistically from $\left(\exists^{2}\right)$ by applying QF-AC ${ }^{1,0}$ to the formula $\forall f^{1} \exists x^{0}(E x=0 \rightarrow f x=0), \neg \neg\left(\exists^{2}\right)$ derives $\neg \neg\left(\mu^{2}\right)$ over $\widehat{\mathrm{WE}_{\mathrm{HA}}}{ }^{\omega} \uparrow+\mathrm{QF}^{-\mathrm{AC}^{1,0}}$. Together with Lemma 4.8, we have

$$
\mathrm{WE}-\mathrm{HA}{ }^{\omega}+\mathrm{QF}-\mathrm{AC}+\mathrm{DC}^{\prime}+\mathrm{M}^{\omega}+\mathrm{BR}+\widetilde{\mathrm{F}^{-}}+\widetilde{\Delta^{\mathscr{M}^{\omega}}} \vdash \neg \neg\left(\mu^{2}\right) .
$$

Applying $\left(\mu^{2}\right)$ to the functions $\lambda k^{0} \cdot(\overline{1, k}),(\overline{1, k})(\mu(\overline{1, k}))=0$ holds for all $k$. Since $\forall k \forall k^{\prime}<k(\overline{1, k})\left(k^{\prime}\right)=1$, $\forall k(\mu(\overline{1, k}) \geq k)$ follows. That is, $\neg \neg \exists \mu \forall k(\mu(\overline{1, k}) \geq k)$, which is intuitionistically equivalent to

$$
\neg \forall \mu \neg \forall k(\mu(\overline{1, k}) \geq k),
$$


follows from $\neg \neg\left(\mu^{2}\right)$. On the other hand, one can easily see that $\mathrm{F}^{-}$(and a-fortiori $\widetilde{\mathrm{F}^{-}}$) derives $\forall \chi^{2} \exists b \forall n \exists z \leq \leq_{0} b(z=\chi(\overline{1, n}))$, and hence $\forall \chi \exists b(\chi(\overline{1, b})<b)$ follows.

Thus, we have

$$
\mathrm{WE}-\mathrm{HA}{ }^{\omega}+\mathrm{QF}-\mathrm{AC}+\mathrm{DC}^{\prime}+\mathrm{M}^{\omega}+\mathrm{BR}+\widetilde{\mathrm{F}^{-}}+\widetilde{\Delta^{\mathscr{M}^{\omega}}} \vdash \perp
$$

Using again Lemma 4.9 applied to $(i i i)$ (note $\widetilde{\widetilde{T}}=\widetilde{\mathrm{T}}$ for $\mathrm{T}$ of the form $\Delta$ ), we have

$$
\mathrm{WE}-\mathrm{HA}{ }^{\omega}+\mathrm{BR}+\widetilde{\mathrm{F}^{-}}+\widetilde{\Delta^{\mathscr{M}^{\omega}}} \vdash \perp .
$$

However, $\mathscr{M}^{\omega}=\mathrm{WE}-\mathrm{HA} \mathrm{A}^{\omega}+\mathrm{BR}+\widetilde{\mathrm{F}^{-}}+\widetilde{\Delta^{\mathscr{M}^{\omega}}}$ follows from the facts $\mathscr{M}^{\omega}=\mathrm{E}-\mathrm{PA}{ }^{\omega}+\mathrm{BR}$ ([19, Theorem 11.17]) and $\mathscr{M}^{\omega} \models \mathrm{F}^{-}$(see [14, Proposition 4.6]) via Lemma 4.10. This completes the proof of Theorem 4.3 in the general case.

In particular, one can take $\Delta^{\mathscr{M}^{\omega}}$ as $\left\{\widetilde{\mathrm{WKL}^{\prime}}\right\}$. To see this, it suffices to note that $\mathscr{M}^{\omega} \models \mathrm{WKL}^{\prime}$ by Lemma 4.10. Since the second-order part $M_{1}$ of $\mathscr{M}^{\omega}$ coincides with the class $S_{1}$ of all functions from natural numbers to natural numbers, $\mathscr{M}^{\omega}$ models WKL. Together with the fact WKL $\leftrightarrow$ WKL ([19, Lemma 9.18.2]), we have $\mathscr{M}^{\omega} \models \mathrm{WKL}^{\prime}$. Therefore, the final assertion of Theorem 4.3 follows from the facts:

- $\widehat{\mathrm{WE}_{\mathrm{H}} \mathrm{HA}}{ }^{\omega} \uparrow \vdash \widetilde{\mathrm{WKL}^{\prime}} \leftrightarrow \operatorname{UWKL}([19$, Lemma 10.32]),

- $\widehat{W E-H A}^{\omega} \uparrow+\mathrm{AC}+\mathrm{WKL} \vdash \mathrm{KL}([19$, Lemma 9.20]),

- $\widehat{\mathrm{WE}-\mathrm{HA}}^{\omega} \uparrow+\mathrm{QF}-\mathrm{AC}^{1,0} \vdash \mathrm{F}^{-} \rightarrow \Sigma_{1}^{0}-\mathrm{UB}^{-}$([19, Proposition 12.6.2]),

- $\mathrm{WE}-\mathrm{HA}^{\omega}+\mathrm{AC}+\mathrm{IP}_{\forall}^{\omega}+\mathrm{M}^{\omega}+\mathrm{BR} \vdash \mathrm{BI}([10$, Theorem $3 B])$.

Remark 4.11. What the proof of Theorem 4.3 actually establishes (together with the simple fact that formulas $B \in \Gamma_{2}$ intuitionistically imply $\left.B^{\prime}\right)$ is that $\mathscr{T}:=\mathrm{WE}-\mathrm{HA}{ }^{\omega}+\mathrm{AC}+\mathrm{IP}_{\forall}^{\omega}+\mathrm{M}^{\omega}+\mathrm{BR}+\mathrm{F}^{-}+\Delta^{\mathscr{M}^{\omega}}$ is consistent but proves $\neg \forall x \exists y A(x, y)$, i.e. not only $\mathscr{T}$ but no consistent extension of $\mathscr{T}$ proves $\forall x \exists y A(x, y)$ (here we use that $\mathrm{DC}^{\prime}$ is - via $\mathrm{BI}$ - derivable in $\mathscr{T}$ ). Note that $\mathrm{F}^{-}$is a classically false principle (in the sense of being inconsistent with $\left(\mu^{2}\right)$ ).

The next corollary is the most useful form of Theorem 4.3 in applications to concrete mathematical principles (note Remark 4.13.2 below).

Corollary 4.12. For a sentence $\mathrm{S}:=\forall x^{\rho}\left(A(x) \rightarrow \exists y^{\tau} B(x, y)\right)$ of $\mathscr{L}\left(\mathrm{E}-\mathrm{H} \mathrm{A}^{\omega}\right)$ where types $\rho, \tau$ are arbitrary, $A(x)$ is purely universal and $B(x, y)$ is in $\Gamma_{2}$, if

$$
\mathrm{WE}-\mathrm{PA}^{\omega}+\mathrm{QF}-\mathrm{AC}+\mathrm{DC}+\mathrm{UWKL}+\mathrm{Uni}(\mathrm{S}) \vdash\left(\exists^{2}\right),
$$

then

$$
\mathrm{WE}-\mathrm{HA}{ }^{\omega}+\mathrm{AC}+\mathrm{IP}_{\forall}^{\omega}+\mathrm{M}^{\omega}+\mathrm{UWKL}+\mathrm{KL}+\Sigma_{1}^{0}-\mathrm{UB}^{-}+\mathrm{BI} \nvdash \mathrm{S} .
$$

Proof. Immediately from Theorem 4.3 since $\mathrm{S}$ is equivalent to the sentence $\forall x \exists y(A(x) \rightarrow B(x, y)) \in \Gamma_{2}$ in the presence of $\mathrm{IP}_{\forall}^{\omega}$. 
Remark 4.13. 1. The previous corollary is false if either $\mathrm{WE}^{\mathrm{PA}}{ }^{\omega}$ is replaced by $\mathrm{E}-\mathrm{PA}{ }^{\omega}$ or $\mathrm{WE}-\mathrm{HA}^{\omega}$ is replaced by $\mathrm{E}-\mathrm{HA}^{\omega}$. One can take $S:=\left(0={ }_{0} 0\right)$ in the first case and take $S:=\left(\exists^{2}\right)$ in the second case, since $\left(\exists^{2}\right)$ is provable in $\widehat{\mathrm{E}-\mathrm{HA}}^{\omega} \uparrow+\mathrm{M}^{\omega}+\mathrm{UWKL}([19$, Corollary 10.62]).

2. The previous corollary does not hold for every sentence $\forall x^{\rho}\left(A(x) \rightarrow \exists y^{\tau} B(x, y)\right)$ in $\Gamma_{2}$. In fact, $\forall f(\exists y(f y=$ $0) \rightarrow \exists x(f x=0)) \in \Gamma_{2}$ is logically valid, but its uniform version $\left(\mu^{2}\right)$ derives $\left(\exists^{2}\right)$ over $\widehat{\mathrm{WE}-\mathrm{HA}}{ }^{\omega} \uparrow$.

Next, as in Proposition 3.15, we show the variant of Corollary 4.12 where WE-HA ${ }^{\omega}$ is replaced by the full extensional system E-HA ${ }^{\omega}$. The remarkable thing in the following corollary is that not only $\Sigma_{1}^{0}$-UB $\mathrm{UB}^{-}$but even $\Sigma_{1}^{0}$-UB is included as well as $\mathrm{BI}^{\leq 1}$ in the extensional semi-intuitionistic system (compare to Proposition 3.15).

Corollary 4.14. For every statement $\mathrm{S}:=\forall x^{\rho}\left(A(x) \rightarrow \exists y^{\tau} B(x, y)\right)$ of $\mathscr{L}\left(\mathrm{E}-\mathrm{HA}{ }^{\omega}\right)$ where $A(x)$ is purely universal, $B(x, y)$ is in $\Gamma_{2}$ and the types of all variables quantified by positively occurring $\forall$ or negatively occurring $\exists$ is not greater than 1 (in particular, $\rho \leq 1$ ), if

$$
\mathrm{WE}-\mathrm{PA}^{\omega}+\mathrm{QF}-\mathrm{AC}+\mathrm{DC}+\mathrm{UWKL}+\mathrm{Uni}(\mathrm{S}) \vdash\left(\exists^{2}\right),
$$

then

$$
\mathrm{E}-\mathrm{HA}{ }^{\omega}+\mathrm{AC} !^{1}+\mathrm{AC}^{0}+\mathrm{IP}_{\forall}^{\leq 1, \leq 1}+\mathrm{M}^{\leq 1}+\mathrm{KL}+\Sigma_{1}^{0}-\mathrm{UB}+\mathrm{BI}^{\leq 1} \nvdash \mathrm{S} .
$$

Proof. Suppose

$$
\mathrm{E}-\mathrm{HA}{ }^{\omega}+\mathrm{AC} !^{1}+\mathrm{AC}^{0}+\mathrm{IP}_{\forall}^{\leq 1, \leq 1}+\mathrm{M}^{\leq 1}+\mathrm{KL}+\Sigma_{1}^{0}-\mathrm{UB}+\mathrm{BI}^{\leq 1} \vdash \mathrm{S} .
$$

$\Sigma_{1}^{0}$-UB follows from $\mathrm{F}$ using $\mathrm{QF}-\mathrm{AC} \mathrm{C}^{1,0}$ (and hence with $\mathrm{AC} !^{1}$ ). Moreover,

$$
\mathrm{E}-\mathrm{HA}{ }^{\omega}+\mathrm{QF}-\mathrm{AC}^{1,0}+\mathrm{M}^{0} \vdash \mathrm{F}^{-} \rightarrow \mathrm{F} .
$$

This follows as in [17, Proposition 3.6] (see also [19, Proposition 12.4]), where this is shown for E-PA ${ }^{\omega}$, since an inspection of the proof shows that only $\mathrm{M}^{0}$ is needed. Hence

$$
\mathrm{E}-\mathrm{HA}^{\omega}+\mathrm{AC} !^{1}+\mathrm{AC}^{0}+\mathrm{IP}_{\forall}^{\leq 1, \leq 1}+\mathrm{M}^{\leq 1}+\mathrm{KL}+\mathrm{F}^{-}+\mathrm{BI}^{\leq 1} \vdash \mathrm{S} .
$$

Since $\mathrm{KL} \leftrightarrow(\mathrm{KL})_{e}, \mathrm{~F}^{-} \rightarrow\left(\mathrm{F}^{-}\right)_{e}$ and $\overline{\mathrm{BI} \leq 1} \rightarrow \overline{\mathrm{BI}^{\leq 1}} e_{e}$ over $\mathrm{WE}-\mathrm{HA}^{\omega}$, as in the proof of Proposition 3.15, one can show

$$
\mathrm{WE}-\mathrm{HA}^{\omega}+\mathrm{AC}+\mathrm{IP}_{\forall}^{\omega}+\mathrm{M}^{\omega}+\mathrm{KL}+\mathrm{F}^{-}+\mathrm{BI}^{\leq 1} \vdash \mathrm{S} .
$$

Therefore the corollary follows from Theorem 4.3 analogously to Corollary 4.12.

We conclude this section by briefly mentioning a variant of Theorem 4.3: The fact that $\exists Y \forall x A(x, Y x)$ classically implies $\left(\exists^{2}\right)$ is usually a reflection of the fact that $\forall x \exists y A(x, y)$ will intuitionistically imply $\Pi_{1}^{0}$-LEM or even $\Sigma_{1}^{0}$-LEM. The latter two principles are not really distinguished in our main theorem as the semi-intuitionistic theory contains Markov's principle by which they are equivalent. Markov's principle is also needed for the negative translation of QF-AC used in the proof to derive $\left(\mu^{2}\right)$ from $\left(\exists^{2}\right)$. However, if $\exists Y \forall x A(x, Y x)$ directly implies $\left(\mu^{2}\right)$ without the use of QF-AC (which usually will be a consequence of $\forall x \exists y A(x, y)$ intuitionistically implying $\Sigma_{1}^{0}$-LEM) then we can draw some additional information about strong semi-intuitionistic theories (not containing $\mathrm{M}^{\omega}$ though) and can allow E-PA ${ }^{\omega}$ instead of WE-PA ${ }^{\omega}$. We don't state the most general result here but just give a sample: 
Proposition 4.15. Let $\forall x^{\rho} \exists y^{\tau} A(x, y)$ be a sentence in $\Gamma_{1}$.

If

$$
\mathrm{E}-\mathrm{PA}^{\omega}+\exists Y \forall x A(x, Y x) \vdash\left(\mu^{2}\right),
$$

then

$$
\mathrm{E}-\mathrm{HA}{ }^{\omega}+\mathrm{AC}+\mathrm{IP}_{\mathrm{ef}}^{\omega}+\mathrm{CA}_{\mathrm{ef}} \nvdash \forall x \exists y A(x, y),
$$

where $\mathrm{CA}_{\mathrm{ef}}$ is the scheme of full comprehension (in all finite types) for $\exists$-free formulas (see [19]).

Note that $\mathrm{CA}_{\mathrm{ef}}$ not only implies UWKL but also e.g.

$$
\left(\tilde{\exists}^{2}\right): \exists E^{2} \forall f^{1}\left(E f=0 \leftrightarrow \forall x^{0}(f x=0)\right) .
$$

Proof. The proof is similar (but simpler) than that of Theorem 4.3 using monotone modified realizability instead of functional interpretation and we only sketch it here. By negative translation applied to the premise, we get

$$
\mathrm{E}-\mathrm{HA}{ }^{\omega}+(\exists Y \forall y A(x, Y x))^{\prime} \vdash\left(\mu^{2}\right)^{\prime}
$$

and so

$$
\mathrm{E}-\mathrm{HA}^{\omega}+(\exists Y \forall y A(x, Y x))^{\prime} \vdash \neg \neg\left(\mu^{2}\right) .
$$

Monotone modified realizability ([19, Theorem 7.1] applied to the negation of the conclusion gives (using that $\left.A \in \Gamma_{1}\right)$

$$
\mathrm{E}-\mathrm{HA}{ }^{\omega}+\mathrm{CA}_{\mathrm{ef}} \vdash \exists Y \forall x A(x, Y x)
$$

and so by negative translation

$$
\mathrm{E}-\mathrm{HA}^{\omega}+\mathrm{CA}_{\mathrm{ef}} \vdash(\exists Y \forall x A(x, Y x))^{\prime} .
$$

Hence

$$
\mathrm{E}-\mathrm{HA}^{\omega}+\mathrm{CA}_{\mathrm{ef}} \vdash \neg \neg\left(\mu^{2}\right)
$$

and so (as in the proof of Theorem 4.3)

$$
\mathrm{E}-\mathrm{HA}^{\omega}+\mathrm{CA}_{\mathrm{ef}}+\mathrm{F}^{-} \vdash \perp
$$

which contradicts

$$
\mathscr{M}^{\omega} \models \mathrm{E}-\mathrm{HA}^{\omega}+\mathrm{CA}_{\mathrm{ef}}+\mathrm{F}^{-} .
$$

\section{Applications}

The uniform versions of the following $\Pi_{2}^{1}$ statements have been investigated in higher order reverse mathematics.

1. WKL. $[16,18,21]$

2. Intermediate value theorem. [18]

3. The attainment of the maximum principle. [18] 
4. Brouwer's fixed point theorem. [18]

5. Weak weak König's lemma WWKL. [21]

6. Bolzano-Weierstraß theorem. [21]

7. Infinite pigeon hole principle RT(1). [21]

Here continuous functions $\Phi:[0,1] \rightarrow \mathbb{R}$ are represented as elements in the Banach space $C[0,1]$ of (equivalence classes of) fast converging (in the uniform norm) sequences of polynomials with rational coefficients (see [22]) which is equivalent to the representation as pairs $\left(\Phi_{r}^{1(0)}, \omega^{1}\right)$ of objects of type degree 1, where $\Phi_{r}$ represents the restriction of $\Phi$ to the dyadic rational numbers in $[0,1]$ and $\omega$ is a modulus of uniform continuity, i.e.

$$
\forall k^{0}, l^{0}, n^{0}\left(\left|r_{k}-\mathbb{Q} r_{l}\right| \leq_{\mathbb{Q}} 2^{-\omega(n)} \rightarrow\left|\Phi_{r} r_{k}-\mathbb{R} \Phi_{r} r_{l}\right| \leq \mathbb{R} 2^{-n}\right)
$$

for some standard enumeration $\left(r_{k}\right)$ of the dyadic rationals in $[0,1]$. Then the premises of 2,3,4 are formalized as purely universal formulas since $\left|\Phi_{r} r_{k}-\mathbb{R} \Phi_{r} r_{l}\right| \leq \mathbb{R} 2^{-n}$ is purely universal. Note that every functional of type 1 represents a real number in Kohlenbach's representation (see [19, Section 4.1] and also [18]). In fact, it is shown in [18] that even the uniform intermediate value theorem for uniformly continuous functions with its modulus derives $\left(\exists^{2}\right)$ over $\mathrm{RCA}_{0}^{\omega}$. Hence this a fortiori is the case for the uniform intermediate value theorem formulated for codes of pointwise continuous functions as in [22]. In the same manner, each of the uniform versions of 3,4 also derives $\left(\exists^{2}\right)$ over $\operatorname{RCA}_{0}^{\omega}[18]$.

For 5,6,7, one has to pay attention to the formalization of uniform versions. A sentence $\forall x\left(\exists u \forall v A_{0}(x, u, v)\right.$ $\rightarrow \exists y B(x, y))$ (like 5.6.7) is intuitionistically equivalent to $\forall x, u\left(\forall v A_{0}(x, u, v) \rightarrow \exists y B(x, y)\right)$. But their uniform versions may have different strength as suggested from [8]. We call the uniform version of the latter one 'strict' uniform version. By inspecting the proofs in [21], one can easily see that each of the strict uniform versions of $5,6,7$ derives $\left(\exists^{2}\right)$ over $R C A_{0}^{\omega}$.

Based on these observations along with the fact that $\left(\exists^{2}\right)$ is not provable in RCA ${ }^{\omega}$, it follows from Proposition 3.4 that all of 1-7 are not provable in $\mathrm{E}-\mathrm{HA}^{\omega}+\mathrm{AC}+\mathrm{IP}_{\mathrm{ef}}^{\omega}+\Delta_{\mathrm{ef}}^{\mathrm{RCA}}{ }^{\omega}$.

Next we turn to discuss some applications of Corollary 4.12 and 4.14. As mentioned in Section 2, RCA ${ }_{0}^{\omega}$ has the full extensionality scheme (E). To show the unprovability of a $\Pi_{2}^{1}$ statement in the strong semi-intuitionistic system via Corollary 4.12 or 4.14 , we have to show that the (strict) uniform version derives $\left(\exists^{2}\right)$ over the weakly extensional system. However, some of the proofs in [21] are carried out still over the weakly extensional version WRCA ${ }_{0}^{\omega}$ (Definition 2.2 in Section 2) of RCA ${ }_{0}^{\omega}$. In fact, the strict uniform versions of Bolzano-Weierstraß theorem or RT $(1)$ derives $\left(\exists^{2}\right)$ over WRCA ${ }_{0}^{\omega}$ respectively, and hence, it follows from Corollary 4.12 and 4.14 that they are provable neither in WE-HA ${ }^{\omega}+\mathrm{AC}+\mathrm{IP}_{\forall}^{\omega}+\mathrm{M}^{\omega}+\mathrm{UWKL}+\mathrm{KL}+\Sigma_{1}^{0}-\mathrm{UB}^{-}+\mathrm{BI}$ nor in $\mathrm{E}-\mathrm{HA}^{\omega}+$ $\mathrm{AC} !^{1}+\mathrm{AC}^{0}+\mathrm{IP}_{\forall}^{\leq 1, \leq 1}+\mathrm{M}^{\leq 1}+\mathrm{KL}+\Sigma_{1}^{0}$-UB $+\mathrm{BI}^{\leq 1}$. On the other hand, it immediately follows from Theorem 4.3 that the uniform version of WWKL, as well as WKL, does not derive $\left(\exists^{2}\right)$ over WRCA ${ }_{0}^{\omega}$ regardless of their formalization (strict or not). One can actually see that the proofs for $1,2,3,4,5$ in $[18,21]$ (i.e. the proofs that their uniform versions imply $\exists^{2}$ ) use the extensionality axioms of type 1(1) or 2 .

Conversely, Corollary 4.14 can be used to show the underivability of $\left(\exists^{2}\right)$ from certain uniform principles over WRCA ${ }_{0}^{\omega}(+\mathrm{UWKL}+\mathrm{DC})$. In fact, one can show that each of $2,3,4$ is provable in $\widehat{\mathrm{E}-\mathrm{HA}}^{\omega} \uparrow+\mathrm{QF}-\mathrm{AC} \mathrm{C}^{0,0}+$ WKL by imitating its uniform proof in $\mathrm{WKL}_{0}$ (see [22]) respectively. Hence it follows via Corollary 4.14 that neither of the uniform versions of 2,3,4 under representing its continuity as uniform continuity with the 
modulus, derives $\left(\exists^{2}\right)$ over WRCA ${ }_{0}^{\omega}$. However, it is still open whether each of the uniform versions of $2,3,4$ in the usual sense of continuity derives $\left(\exists^{2}\right)$ over $\mathrm{WRCA}_{0}^{\omega}(+\mathrm{UWKL}+\mathrm{DC})$.

For the purpose of demonstrating that our metatheorem is extensively applicable to statements which are provable in $\mathrm{RCA}_{0}$ but whose sequential versions derive ACA, we shall treat the following principles studied in preceding papers.

1. Jordan decomposition for $2 \times 2$ matrices.

2. Trichotomy for reals.

3. $\Pi_{1}^{0}$ least number principle.

In the following, we see that each of them has a syntactical form to which Corollary 4.12 and 4.14 are applicable, and that each of their uniform versions derives $\left(\exists^{2}\right)$ over WRCA ${ }_{0}^{\omega}$ (reflecting the fact that the pointwise versions intuitionistically imply $\Pi_{1}^{0}$-LEM or even $\Sigma_{1}^{0}$-LEM). The proofs are similar to those in [21]. At first, we consider the Jordan decomposition for $2 \times 2$ matrices. As shown in [9, Section 4], it is provable in RCA 0 but its sequential

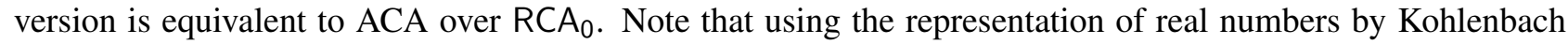
[19, Section 4.1], every functional of type 1 can be seen to represent a unique real number. Furthermore, since a complex number is naturally defined as a pair of real numbers, every functional of type 1 also represents a $2 \times 2$ complex matrix via the standard encoding.

Definition 5.1 (See [9] for details).

$\mathrm{JD}_{2}: \quad \forall M^{1}\left(M\right.$ is a $2 \times 2$ complex matrix $\rightarrow \exists U^{1}, J^{1}$

$\left(U, J\right.$ are $2 \times 2$ complex matrices such that $M=U J U^{-1}$ and $J$ consists of Jordan blocks $\left.)\right)$.

Note that the tuple of $U^{1}$ and $J^{1}$ can be coded as a single variable of type 1 .

Theorem 5.2. $W R C A_{0}^{\omega}+\operatorname{Uni}\left(\mathrm{JD}_{2}\right) \vdash\left(\exists^{2}\right)$.

Proof. We reason in $\mathrm{WRCA}_{0}^{\omega}$. By primitive recursion with a parameter of type 1, define a functional $\Xi$ of type $1 \rightarrow 1$ such that

$$
\Xi(f)(m):= \begin{cases}\frac{1}{2^{n_{l}+1}} \text { where } n_{l} \text { is a least number such that } f\left(n_{l}\right)=0 & \text { if } \exists n \leq m f(n)=0, \\ \frac{1}{2^{m+1}} & \text { otherwise. }\end{cases}
$$

Then for every $f$ of type $1, \Xi(f)$ represents a real number. Furthermore, there exists $n$ such that $f(n)=0$ if and only if $\Xi(f) \neq \mathbb{R} 0$. By a standard discussion as in linear algebra, one can show that for every $x \in \mathbb{C}$, the Jordan canonical form of $\left(\begin{array}{ll}1 & 0 \\ x & 1\end{array}\right)$ is $\left(\begin{array}{ll}1 & 1 \\ 0 & 1\end{array}\right)$ if $x \neq \mathbb{C} 0$, and $\left(\begin{array}{ll}1 & 0 \\ 0 & 1\end{array}\right)$ if $x=\mathbb{C} 0$. As mentioned in the proof of [9, Lemma 4.4], it is effectively decided whether the upper right-hand entry of the Jordan canonical form of $\left(\begin{array}{ll}1 & 0 \\ \Xi(f) & 1\end{array}\right)$ is 0 or 1 . Then $\exists n(f(n)=0)$ is equivalent to some quantifier-free formula with $\Xi$, so by [19, Proposition 3.17], one can construct a term $t$ of type 2 such that $t(f)=0 \leftrightarrow \exists n(f(n)=0)$ for every $f$. Therefore we have $\left(\exists^{2}\right)$.

Corollary 5.3. $\mathrm{JD}_{2}$ is provable neither in $\mathrm{WE}_{-} \mathrm{HA}^{\omega}+\mathrm{AC}+\mathrm{IP}_{\forall}^{\omega}+\mathrm{M}^{\omega}+\mathrm{UWKL}+\mathrm{KL}+\Sigma_{1}^{0}-\mathrm{UB}^{-}+\mathrm{BI}$ nor in $\mathrm{E}-\mathrm{HA}{ }^{\omega}+\mathrm{AC}^{1}+\mathrm{AC}^{0}+\mathrm{IP}_{\forall}^{\leq 1, \leq 1}+\mathrm{M}^{\leq 1}+\mathrm{KL}+\Sigma_{1}^{0}-\mathrm{UB}+\mathrm{BI}^{\leq 1}$. 
Proof. This follows immediately from the previous theorem applied to Corollary 4.12 and 4.14 (note that ' $M$ is a $2 \times 2$ complex matrix' is dropped in Kohlenbach's representation and the conclusion of $\mathrm{JD}_{2}$ is in $\Gamma_{2}$ ).

Remark 5.4. $\quad$ 1. The above corollary extends the unprovability result mentioned at the end of Hirst and Mummert [9].

2. The Jordan canonical form depends on the eigenvalues of the given matrix. As mentioned in [4], the fundamental theorem of algebra is provable in $\mathrm{E}-\mathrm{HA}{ }^{\omega}+\mathrm{QF}-\mathrm{AC}{ }^{0,0}+\mathrm{WKL}$. That is, one can show that every complex matrix has complex eigenvalues within $\mathrm{E}-\mathrm{HA}^{\omega}+\mathrm{QF}-\mathrm{AC}^{0,0}+\mathrm{WKL}$ but cannot construct its Jordan canonical form even in $\mathrm{E}-\mathrm{HA}{ }^{\omega}+\mathrm{AC} !^{1}+\mathrm{AC}^{0}+\mathrm{IP}_{\forall}^{\leq 1, \leq 1}+\mathrm{M}^{\leq 1}+\mathrm{KL}+\Sigma_{1}^{0}-\mathrm{UB}+\mathrm{BI} \leq 1$.

Next, we consider the trichotomy for reals. As shown in [5, Section 2], it is provable in $\mathrm{RCA}_{0}$ but its sequential version is equivalent to ACA over $\mathrm{RCA}_{0}$. Note that $\alpha^{1} \geq_{\mathbb{R}} \beta^{1}$ and $\alpha^{1}=_{\mathbb{R}} \beta^{1}$ are represented as purely universal formulas and $\alpha^{1}>_{\mathbb{R}} \beta^{1}$ is represented as a purely existential formula.

\section{Definition 5.5.}

$$
\begin{gathered}
\text { LPO }: \forall f^{1}(\forall n(f(n) \leq 1) \rightarrow(\exists n(f(n)=1) \vee \forall n(f(n)=0))) . \\
\text { TRI }: \forall \alpha^{1}\left(\alpha \in \mathbb{R} \rightarrow \alpha>_{\mathbb{R}} 0 \vee \alpha=_{\mathbb{R}} 0 \vee \alpha<_{\mathbb{R}} 0\right) . \\
\text { TRI }^{-}: \forall \alpha^{1}\left(\alpha \in \mathbb{R} \wedge \alpha \geq_{\mathbb{R}} 0 \rightarrow \alpha>_{\mathbb{R}} 0 \vee \alpha=_{\mathbb{R}} 0\right) .
\end{gathered}
$$

Here we think of $A \vee B$ as an abbreviation of $\exists k((k=0 \rightarrow A) \wedge(k \neq 0 \rightarrow B))$. Note that $\alpha \in \mathbb{R}$ is dropped in Kohlenbach's [19] representation (in which every $\alpha^{1}$ represents a unique real number).

Theorem 5.6. $\mathrm{WRCA}_{0}^{\omega}+\operatorname{Uni}\left(\mathrm{TRI}^{-}\right) \vdash\left(\exists^{2}\right)$.

Proof. We reason in $\mathrm{WRCA}_{0}^{\omega}$. By primitive recursion with a parameter of type 1, define a functional $\Xi$ of type $1 \rightarrow 1$ as in the proof of Theorem 5.2. Then for every $f$ of type $1, \Xi(f)$ is a real number and $\Xi(f) \geq \mathbb{R} 0$. Let $\Psi$ be a witness of Uni(TRI $\left.{ }^{-}\right)$. One can easily show $\exists n f(n)=0 \leftrightarrow \Psi(\Xi(f)) \neq 0$. Note that the right side in this equivalence is a quantifier-free formula. Then we have $\left(\exists^{2}\right)$.

Since ' $\alpha \geq_{\mathbb{R}} 0$ ' is purely universal and the proof of [5, Theorem 1] shows LPO $\leftrightarrow \mathrm{TRI} \leftrightarrow \mathrm{TRI}^{-}$over $\widehat{\mathrm{WE}-\mathrm{HA}}{ }^{\omega} \uparrow+$ $\mathrm{QF}-\mathrm{AC}^{0,0}$, the next corollary immediately follows as before.

Corollary 5.7. Each of $\mathrm{LPO}$, TRI and $\mathrm{TRI}^{-}$is provable neither in $\mathrm{WE}^{-\mathrm{HA}^{\omega}}+\mathrm{AC}+\mathrm{IP}_{\forall}^{\omega}+\mathrm{M}^{\omega}+\mathrm{UWKL}+$ $\mathrm{KL}+\Sigma_{1}^{0}-\mathrm{UB}^{-}+\mathrm{BI}$ nor in $\mathrm{E}-\mathrm{HA}^{\omega}+\mathrm{AC}^{1}+\mathrm{AC}^{0}+\mathrm{IP}_{\forall}^{\leq 1, \leq 1}+\mathrm{M} \leq 1+\mathrm{KL}+\Sigma_{1}^{0}-\mathrm{UB}+\mathrm{BI} \leq 1$.

At the end, we consider the least number principle with a parameter of type 1 . In the practice of reverse mathematics, the least number principle often appears in non-uniform proofs within RCA. It is known that $\Pi_{n}^{0}$-least number principle is equivalent to $\Sigma_{n}^{0}$-induction over $\mathrm{RCA}_{0}^{*}$. On the other hand, one can show that the sequential version of $\Pi_{1}^{0}$ least number principle with a set parameter is equivalent to ACA over RCA $A_{0}$ using the idea of the proof of Theorem 5.9 below.

\section{Definition 5.8.}

$$
\mathrm{L}_{1}^{0}(\varphi): \forall x^{0}, g^{1}\left(\varphi(x, g) \rightarrow \exists x_{l}^{0}\left(\varphi\left(x_{l}, g\right) \wedge \forall x^{\prime}<x_{l} \neg \varphi\left(x^{\prime}, g\right)\right)\right),
$$

where $\varphi$ is a $\Pi_{1}^{0}$-formula which may have more parameters. 
Theorem 5.9. Let $\varphi_{1}(x, \alpha)$ be the $\Pi_{1}^{0}$-formula expressing $\alpha \leq_{\mathbb{R}}\langle x\rangle_{i \in \mathbb{N}}$ where $\langle x\rangle_{i \in \mathbb{N}}$ denotes the infinite constant$x$ sequence. Then $\mathrm{WRCA}_{0}^{\omega}+\operatorname{Uni}\left(\operatorname{L\Pi }_{1}^{0}\left(\varphi_{1}\right)\right) \vdash\left(\exists^{2}\right)$.

Proof. By primitive recursion with a parameter of type 1 , define a functional $\Xi$ of type $1 \rightarrow 1$ as in the proof of Theorem 5.2. Then for every $f$ of type $1, \Xi(f) \leq \mathbb{R} 1$ holds. Let $\Psi$ be a witness of Uni $\left(\operatorname{L\Pi }_{1}^{0}\left(\varphi_{1}\right)\right)$. One can easily show $\exists n f(n)=0 \leftrightarrow \Psi(\Xi(f)) \neq 0$. Hence we can take $E(f):=\overline{s g}(\Psi(\Xi(f)))$ to derive $\left(\exists^{2}\right)$.

Corollary 5.10. $\mathrm{L}_{1}^{0}$ is provable neither in $\mathrm{WE}-\mathrm{HA}^{\omega}+\mathrm{AC}+\mathrm{IP}_{\forall}^{\omega}+\mathrm{M}^{\omega}+\mathrm{UWKL}+\mathrm{KL}+\Sigma_{1}^{0}-\mathrm{UB}^{-}+\mathrm{BI}$ nor in $\mathrm{E}-\mathrm{HA}{ }^{\omega}+\mathrm{AC}^{1}+\mathrm{AC}^{0}+\mathrm{IP}_{\forall}^{\leq 1, \leq 1}+\mathrm{M}^{\leq 1}+\mathrm{KL}+\Sigma_{1}^{0}-\mathrm{UB}+\mathrm{BI}^{\leq 1}$.

\section{Acknowledgment}

The first author is grateful to Takeshi Yamazaki for helpful discussion.

\section{References}

[1] Y. Akama, S. Berardi, S. Hayashi and U. Kohlenbach, An arithmetical hierarchy of the law of excluded middle and related principles. Proc. of the 19th Annual IEEE Symposium on Logic in Computer Science (LICS'04), pp. 192-201, IEEE Press (2004).

[2] J. Avigad and S. Feferman, Gödel's functional ('Dialectica') interpretation. In: S. R. Buss (Ed.), Handbook of Proof. Theory, Elsevier Science B.V., Amsterdam, 1998, pp. 337-410.

[3] M. A. Bezem, Strongly majorizable functionals of finite type: a model for bar recursion containing discontinuous functionals. J. Symb. Logic 50, 652-660 (1985).

[4] F. G. Dorais, Classical consequences of continuous choice principles from intuitionistic analysis, Notre Dame Journal of Formal Logic, to appear.

[5] F. G. Dorais, J. L. Hirst and P. Shafer, Reverse mathematics, trichotomy, and dichotomy, Journal of Logic and Analysis 4(13), (2012) 1-14.

[6] S. Feferman, Theories of finite type related to mathematical practice, in J. Barwise (ed.) Handbook of Mathematical Logic, North-Holland, Amsterdam, 1977, 913-971.

[7] F. Ferreira, Proof interpretations and majorizability. In: Delon, F. et al (eds.), Logic Colloquium 2007, Lecture Notes in Logic 35, Cambridge University Press 2010, pp. 32-81.

[8] M. Fujiwara and K. Yokoyama, A note on the sequential version of $\Pi_{2}^{1}$ statements, Lecture Notes in Computer Science vol. 7921 (2013), pp. 171-180.

[9] J. L. Hirst and C. Mummert, Reverse mathematics and uniformity in proofs without excluded middle, Notre Dame Journal of Formal Logic 52 (2011), no.2, pp. 149-162.

[10] W. A. Howard, Functional interpretation of bar induction by bar recursion. Compositio Mathematica, 20: pp. 107-124, 1968. 
[11] W. A. Howard and G. Kreisel, Transfinite induction and bar induction of types zero and one, and the role of continuity in intuitionistic analysis, Journal of Symbolic Logic, 31(3): pp. 325-358, 1966.

[12] U. Kohlenbach, Pointwise hereditary majorization and some applications, Archive for Mathematical Logic, vol. 31, 1992, pp. 227-241.

[13] U. Kohlenbach, Effective bounds from ineffective proofs in analysis: an application of functional interpretation and majorization. Journal of Symbolic Logic, vol. 57, 1992, pp. 1239-1273.

[14] U. Kohlenbach, Mathematically strong subsystems of analysis with low rate of growth of provably recursive functionals, Archive for Mathematical Logic, vol. 36, 1996, pp. 31-71.

[15] U. Kohlenbach, A note on Spector's quantifier-free rule of extensionality, Archive for Mathematical Logic 40, pp. 89-92, 2001.

[16] U. Kohlenbach, On uniform weak König's lemma, Annals of Pure and Applied Logic 114, pp. 103-116, 2002.

[17] U. Kohlenbach, Foundational and mathematical uses of higher types. In: W. Sieg et al. (eds.), Reflections on the foundations of mathematics. Lecture Notes in Logic 15, Assoc. Symbol. Logic and A.K. Peters, pp. 92-116.

[18] U. Kohlenbach, Higher order reverse mathematics in Reverse mathematics 2001, S. Simpson ed., pp. 281-295, Lecture Notes in Logic 21, Assoc. Symbol. Logic and A. K. Peters, Wellesley MA 2005.

[19] U. Kohlenbach, Applied proof theory: Proof Interpretations and their Use in Mathematics, Springer Monographs in Mathematics, Springer-Verlag, Berlin, 2008.

[20] H. Luckhardt, Extensional Gödel Functional Interpretation. Springer Lecture Notes in Mathematics 306, 1973.

[21] N. Sakamoto and T. Yamazaki, Uniform versions of some axioms of second order arithmetic, Mathematical Logic Quarterly 50(6) (2004) pp. 587-593.

[22] S. G. Simpson, Subsystems of Second Order Arithmetic, Second Edition, Association for Symbolic Logic. Cambridge University Press, 2009.

[23] C. Spector, Provably recursive functionals of analysis: a consistency proof of analysis by an extension of principles in current intuitionistic mathematics. In F. D. E. Dekker, editor, Recursive Function Theory: Proceedings of Symposia in Pure Mathematics, volume 5, pp. 1-27. American Mathematical Society, Providence, Rhode Island, 1962.

[24] A. S. Troelstra (ed.), Metamathematical Investigation of Intuitionistic Arithmetic and Analysis, Lecture notes in Mathematics, Vol. 344, Springer-Verlag, Berlin, 1973. 